\title{
Dynamic martensitic phase transformation in single-crystal silver microcubes
}

Thevamaran, Ramathasan; Griesbach, Claire; Yazdi, Sadegh; Ponga, Mauricio; Alimadadi, Hossein; Lawal, Olawale; Jeon, Seog Jin; Thomas, Edwin L.

Published in:

Acta Materialia

Link to article, DOI:

10.1016/j.actamat.2019.10.006

Publication date:

2020

Document Version

Peer reviewed version

Link back to DTU Orbit

Citation (APA):

Thevamaran, R., Griesbach, C., Yazdi, S., Ponga, M., Alimadadi, H., Lawal, O., Jeon, S. J., \& Thomas, E. L. (2020). Dynamic martensitic phase transformation in single-crystal silver microcubes. Acta Materialia, 182, 131 143. https://doi.org/10.1016/j.actamat.2019.10.006

\section{General rights}

Copyright and moral rights for the publications made accessible in the public portal are retained by the authors and/or other copyright owners and it is a condition of accessing publications that users recognise and abide by the legal requirements associated with these rights.

- Users may download and print one copy of any publication from the public portal for the purpose of private study or research.

- You may not further distribute the material or use it for any profit-making activity or commercial gain

- You may freely distribute the URL identifying the publication in the public portal

If you believe that this document breaches copyright please contact us providing details, and we will remove access to the work immediately and investigate your claim 


\section{Journal Pre-proof}

Dynamic Martensitic Phase Transformation in Single-crystal Silver Microcubes

Ramathasan Thevamaran, Claire Griesbach , Sadegh Yazdi , Mauricio Ponga, Hossein Alimadadi, Olawale Lawal, Seog-Jin Jeon , Edwin L. Thomas

PII:

DOI:

Reference:

To appear in:

Received date:

Revised date:

Accepted date:
S1359-6454(19)30666-4

https://doi.org/10.1016/j.actamat.2019.10.006

AM 15574

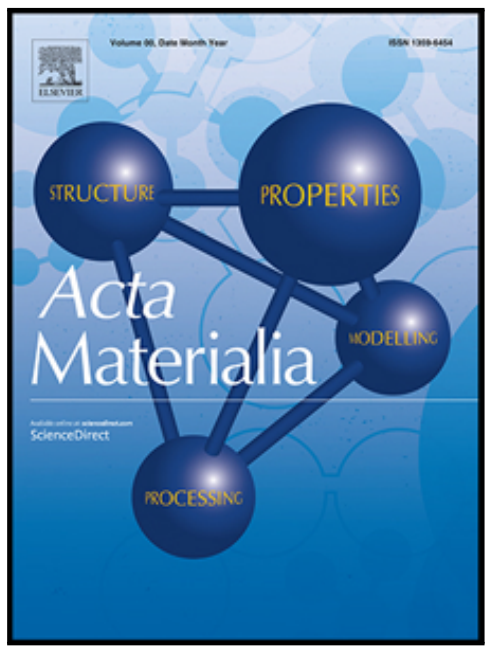

Please cite this article as: Ramathasan Thevamaran, Claire Griesbach, Sadegh Yazdi, Mauricio Ponga, Hossein Alimadadi, Olawale Lawal, Seog-Jin Jeon, Edwin L. Thomas, Dynamic Martensitic Phase Transformation in Single-crystal Silver Microcubes, Acta Materialia (2019), doi: https://doi.org/10.1016/j.actamat.2019.10.006

This is a PDF file of an article that has undergone enhancements after acceptance, such as the addition of a cover page and metadata, and formatting for readability, but it is not yet the definitive version of record. This version will undergo additional copyediting, typesetting and review before it is published in its final form, but we are providing this version to give early visibility of the article. Please note that, during the production process, errors may be discovered which could affect the content, and all legal disclaimers that apply to the journal pertain.

(C) 2019 Acta Materialia Inc. Published by Elsevier Ltd. All rights reserved. 


\title{
Dynamic Martensitic Phase Transformation in Single- crystal Silver Microcubes
}

Ramathasan Thevamaran ${ }^{1, *}$, Claire Griesbach ${ }^{1}$, Sadegh Yazdi ${ }^{2}$, Mauricio Ponga ${ }^{3}$, Hossein Alimadadi $^{4,5}$, Olawale Lawal ${ }^{6}$, Seog-Jin Jeon ${ }^{7}$, Edwin L. Thomas, ${ }^{6, *}$.

${ }^{I}$ Department of Engineering Physics, University of Wisconsin-Madison, Madison, WI 53706, USA.

${ }^{2}$ Renewable and Sustainable Energy Institute, University of Colorado-Boulder, Boulder, CO 80309, USA.

${ }^{3}$ Department of Mechanical Engineering, University of British Columbia, Vancouver, BC V6T 1Z4,

Canada.

${ }^{4}$ DTU Danchip/Cen, Technical University of Denmark, Fysikvej, Building 307, DK-2800 Kgs. Lyngby, Denmark.

${ }^{5}$ Danish Technological Institute, Kongsvang Allé 29, 8000 Aarhus C, Denmark.

${ }^{6}$ Department of Materials Science and NanoEngineering, Rice University, Houston, TX 77025, USA.

${ }^{7}$ Department of Polymer Science and Engineering, Kumoh National Institute of Technology, Gumi, Gyoeongbuk 39177, South Korea.

*Corresponding Authors: thevamaran@wisc.edu (RT); $\underline{\text { elt@ rice.edu }}$ (ELT)

\begin{abstract}
The ability to transform the crystal structure of metals in the solid state enables tailoring their physical, mechanical, electrical, thermal, and optical properties in unprecedented ways. We demonstrate a martensitic phase transformation from a face-centered-cubic (fcc) structure to a hexagonal-close-packed (hcp) structure that occurs in nanosecond timescale in initially near-defectfree single-crystal silver (Ag) microcubes impacted at supersonic velocities. Impact-induced high pressure and high strain rates in Ag microcubes cause impact orientation dependent extreme microand nano-structural transformations. When a microcube is impacted along the [100] crystal symmetry direction, the initial fcc structure transforms into an hep crystal structure, while impact along the [110] direction does not produce phase transformations, suggesting the predominant role played by the stacking faults generated in the [100] impact. Molecular dynamics simulations at
\end{abstract}


comparable high strain rates reveal the emergence of such stacking faults that coalesce, forming large hcp domains. The formation of hcp phase through the martensitic transformation of fcc $\mathrm{Ag}$ shows new potential to dramatically improve material properties of low-stacking-fault energy materials.

\section{Introduction}

Martensitic phase transformation - a stress or thermally-induced non-diffusive solid-state phase transformation - that results in a change of crystal structure has been found in many material systems such as shape memory and structural alloys [1-4], and can occur during meteorite impacts [5]. Because of the drastic changes in microstructure, the martensitic phase transformation has often been exploited as a useful tool to achieve desirable active control of material properties - e.g., biomedical shape memory implants that utilize the martensitic phase transformation in NiTi alloys[2,6] - and to improve mechanical properties through the creation of specific desirable microstructures - e.g. synthesis of hexagonal and cubic diamond from graphite $[7,8]$ and cubic and zincblend boron nitrides from rhombohedral boron nitride [9]. Crystallographic structural changes induced by phase transformation has also been found in pure metals such as bulk titanium and tantalum, as well as silver nanoparticles [10-13].

Silver (Ag) is a low-stacking-fault energy noble metal that is most commonly found in the face-centered-cubic (fcc) structure (lattice constant $\sim 0.408 \mathrm{~nm}$ ) that belongs to the space group $F m \overline{3} m$ (No. 225) [14]. The closed packed atomic planes in an fcc crystal are arranged in an ABC stacking sequence along the [111] crystallographic direction (also denoted as $3 \mathrm{C}$ phase). $\mathrm{Ag}$ is added to high-temperature superconductor wires, tapes, and films to shield the superconducting material from its surroundings while providing mechanical strength and internal strain-relief for the brittle superconductor, stabilization of the superconductor through alternate current path and thermal path to the coolant, and low-resistance electrical contact [14]. Ag also exhibits high thermal and electrical conductivities, diamagnetism, high reflectance, and low emittance [14]. Ag has attracted attention recently for plasmonic applications as well-for example, use of Ag nanoparticles for enhanced trapping of light in solar cells - because of their large surface enhancement factors and localized surface plasmon resonance that can be tuned to visible and infrared wavelengths through shape modifications [15]. Due to their highest reflectivity among metals, colloidal Ag particles have also been proposed for coating space mirrors that can be used for detecting even the faintest signals 
from far distances [16]. The ability to dramatically modify the structure and the properties of $\mathrm{Ag}$ micro- and nanoparticles while simultaneously improving their strength and toughness can greatly benefit these futuristic applications of Ag.

Though it is rare, naturally occurring hexagonal-close-packed (hcp) Ag (space group: $\mathrm{P}_{3} / \mathrm{mmc}($ No. 194)) in both $4 \mathrm{H}$ (lattice constants $\mathrm{a}=0.288 \mathrm{~nm}, \mathrm{c}=1.0 \mathrm{~nm}, \mathrm{c} / \mathrm{a}=3.47$ ) and $2 \mathrm{H}$ (lattice constants $\mathrm{a}=0.283 \mathrm{~nm}, \mathrm{c}=0.638 \mathrm{~nm}, \mathrm{c} / \mathrm{a}=2.25$ ) polytypes has also been found previously as inter-grown layers in fcc Ag in the native silver-gold ores of north-eastern Russia [17]. These metastable formations could have occurred due to the slow growth of individual crystals and could have been stabilized due to admixtures such as antimony ( $\mathrm{Sb}$ ), bismuth $(\mathrm{Bi})$, copper $(\mathrm{Cu})$, and iron (Fe) [17]. A few atomic layers thick $4 \mathrm{H} \mathrm{Ag}$ has also been synthesized using epitaxial growth process [18]. The $4 \mathrm{H} \mathrm{Ag}$ has recently been found in nanocrystalline (grain sizes $<30 \mathrm{~nm}$ ) Ag thinfilms fabricated using high-pressure magnetron sputtering [19]. 4H Ag has also been synthesized in nanorods (diameter $\sim 10-100 \mathrm{~nm}$ ) [20-23], nanoribbons [24] and bulk films [25-27]. Bulk 2H Ag has also been synthesized [25,26] using electrochemical deposition, though it is extremely rare due to its highly metastable nature and as it can spontaneously transform to the fcc phase at room temperature [27]. The $4 \mathrm{H}$ phase is more stable than the $2 \mathrm{H}$ phase, but it also undergoes electronbeam-radiation-induced [23] and thermally-induced [27] phase transformations from $4 \mathrm{H}$ to $3 \mathrm{C}$.

Here, we demonstrate that the initial single-crystal fcc structure of $\mathrm{Ag}$ can be transformed into a gradient-nano-grained hcp structure through high-velocity impact of the Ag microcubes along a specific intrinsic crystal symmetry direction. Molecular dynamics simulations support experimental evidence of an fcc to hcp phase transformation and provide insights into the atomistic deformation mechanisms that lead to the phase transformation. Additionally, we investigate the stability of the hcp phase through temporal observational studies.

\section{Materials and methods}

\subsection{Experimental setup}

\subsubsection{Silver micro-cube synthesis}

We synthesized monodisperse $\sim 1.4 \mu \mathrm{m}$ Ag cubes (Fig.1a) using a multistep seed-growth process in a controlled atmosphere [28,29]. Ag nanocubes that are used as seed for Ag microcube growth were synthesized in a controlled reaction atmosphere as detailed in the paper by Jeon et al. [29]. Typically, $1 \mathrm{ml}$ of ethylene glycol ( $\mathrm{EG}, \mathrm{Cl} \leq 0.2 \mathrm{mg} / \mathrm{kg}$ and $\mathrm{Fe} \leq 0.2 \mathrm{mg} / \mathrm{kg}$, Aldrich) is preheated at $140^{\circ} \mathrm{C}$ while stirring at $\sim 900 \mathrm{rpm}$ in a $22 \mathrm{ml}$ vial for 10 minutes. $50 \mu \mathrm{l}$ of $60 \mathrm{mM}$ 
hydrochloric acid ( $\mathrm{HCl}, 37 \%$, Aldrich) solution in EG is added into the vial. After 2 minutes, $1 \mathrm{ml}$ of $0.2 \mathrm{M}$ silver nitrate $\left(\mathrm{AgNO}_{3}\right.$, Aldrich) in $\mathrm{EG}$ injected in drops into the vial. Three minutes later, 1 $\mathrm{ml}$ of $0.12 \mathrm{M}$ polyvinylpyrrolidone (PVP, MW 55,000, Aldrich) solution in EG is added in drops. 4 hours after the addition of PVP solution, the vial is capped. Seeding starts in an hour as indicated by a yellowish color. Growth is completed in another 35 minutes. The reaction batch is thermally quenched in a water bath at room temperature for 20 minutes. The seed nano-cubes are washed with acetone once, followed by several water washes to remove EG and PVP. Finally, the seed nanocubes are dispersed in $1 \mathrm{ml}$ of EG for further growth.

The first seed growth under Ar atmosphere is performed as described in the papers by Jeon et al. [28]. Typically, $3 \mathrm{ml}$ of $\mathrm{EG}$ is preheated at $160^{\circ} \mathrm{C}$ for 10 minutes with Ar introduction (Matheson) and $60 \mu \mathrm{l}$ of $600 \mathrm{mM} \mathrm{HCl}$ solution is added into the vial. A glass pipet is used to introduce a continuous flow of Ar gas above the reaction solution. After 2 minutes, $1 \mathrm{ml}$ of $0.8 \mathrm{M}$ $\mathrm{AgNO}_{3}$ solution is injected in drops into the vial. After another 2 minutes, $1 \mathrm{ml}$ of $0.4 \mathrm{M}$ PVP solution is added in drops. A $120 \mu \mathrm{l}$ of seed suspension is injected 3 minutes after the PVP injection. After another 35 minutes, the reaction vial is quenched in room temperature water. The product is washed with acetone once, followed by several water washes, and filtered to remove small portion of nano-wires $(\sim 5 \%)$. Finally, filtered particles are dispersed in $1 \mathrm{ml}$ of EG for further seed growth.

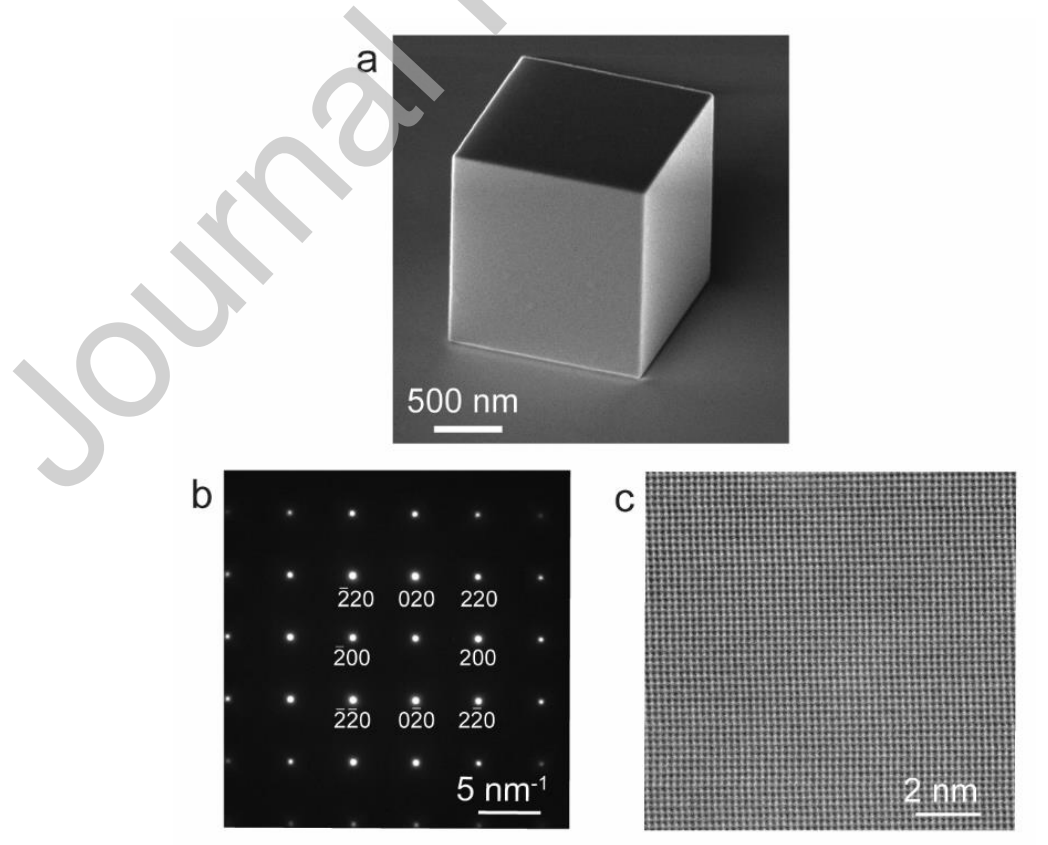

Figure 1. Scanning electron microscopy (SEM), selective area diffraction (SAD), and high-resolution transmission electron microscopy (HR-TEM) images of a representative near-defect-free single-crystal Ag microcube 
The second seed growth under Ar atmosphere is performed as described in the papers by Jeon et al. [28,29]. A $3 \mathrm{ml}$ of EG is preheated at $160^{\circ} \mathrm{C}$ for 10 minutes under Ar introduction, and 90 $\mu \mathrm{l}$ of $600 \mathrm{mM} \mathrm{HCl}$ solution is added into the vial. After 2 minutes, $1 \mathrm{ml}$ of $1.6 \mathrm{M} \mathrm{AgNO}_{3}$ solution is injected in drops into the vial. 2 minutes later, $1 \mathrm{ml}$ of $0.8 \mathrm{M} \mathrm{PVP}$ solution in EG is added in drops. $170 \mu \mathrm{l}$ of particles synthesized in first seed growth (step b) are injected 3 minutes after the PVP injection. Another 35 minutes later, the reaction vial is quenched in room temperature water and the product is washed with acetone once, followed by several water washes, and filtered to remove small portion of nanowires. The final product, Ag micro-cubes, are dispersed in $1 \mathrm{ml}$ of distilled water for storage.

Selective area diffraction (SAD) and high-resolution transmission electron microscope (HRTEM) images of a thin lamella obtained using focused ion beam (FIB) milling from the assynthesized microcubes show that the samples are near-defect-free single-crystals (Fig.1b-c).

\subsubsection{Impact testing}

We use an advanced laser-induced projectile impact testing (LIPIT) apparatus [30,31] to launch the Ag microcubes at a supersonic velocity of $\sim 400 \mathrm{~ms}^{-1}$ and impact them onto impenetrable rigid targets (Fig.2a). The LIPIT apparatus consists of four main components: (i) an Nd: YAG pulse laser $(\lambda=1064 \mathrm{~nm})$ and optical components to launch the projectile by laser ablation of an underlying Au layer, (ii) launch pad - a glass substrate that is coated first with a $\sim 50 \mathrm{~nm}$ gold (Au) film and then a $\sim 20 \mu \mathrm{m}$ crosslinked polydimethylsiloxane (PDMS) layer, in which the Au-layer absorbs laser energy from a nanosecond pulse and vaporizes causing the rubbery PDMS to rapidly expand and launch the projectile at velocities proportional to the laser energy, (iii) rigid target - a silicon substrate coated with $\sim 100 \mathrm{~nm}$ gold film, and (iv) in-situ imaging of the projectile in-flight by a CCD camera with microscopic lens that is illuminated at $34.5 \mathrm{~ns}$ intervals by two incoherent light pulses that are generated by amplified spontaneous emission from a dye laser cavity with a gain medium (Rhodamine 640 in ethanol), which is optically pumped from another Nd: YAG laser $(\lambda=532 \mathrm{~nm})$ (time delay between pulses is achieved by an optical delay path in air). The apparatus is also equipped with another CCD camera with microscopic lens and LED illumination to visualize and align the projectile to the focal point of the ablation laser on the launch pad. 


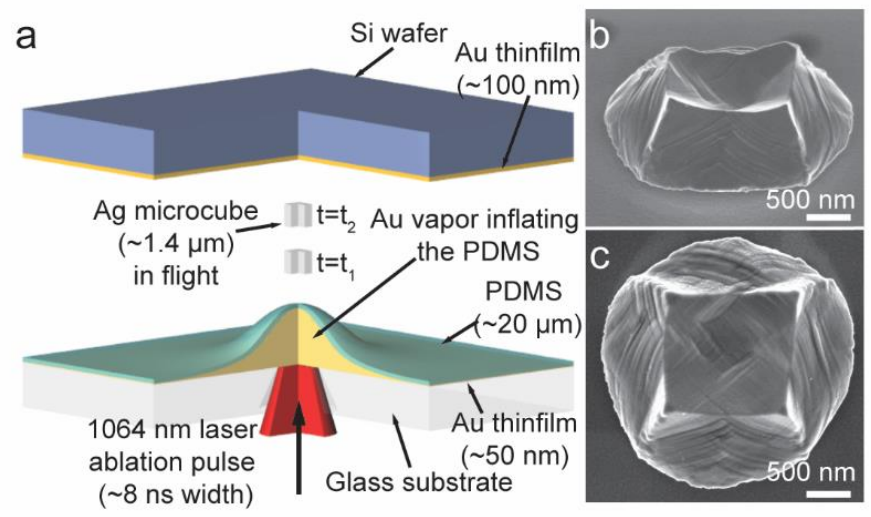

Figure 2. (a) An illustration of the LIPIT, (b) post-impact side (at $52^{\circ}$ tilt) SEM view of an Ag microcube, and (c) post-impact top SEM view of an Ag microcube

The Ag micro-cubes preserved in ethanol are drop-cast on top of the PDMS layer and airdried on the launch pad before LIPIT. The PDMS layer prevents sample heating from the laser ablation of $\mathrm{Au}$, in addition to launching the projectile. Additionally, the Au layer thickness has been chosen to suppress (below 1\%) optical transmission of the laser pulse energy during ablation.

The ultra-high-strain-rate $\left(\sim 10^{8} \mathrm{~s}^{-1}\right)$ deformation produces a unique gradient-nano-grained (GNG) structure - with the grain size varying from $\sim 5 \mathrm{~nm}$ near the impacted bottom region of the sample to $\sim 500 \mathrm{~nm}$ at the top region of the sample - through the interplay between a hydrodynamic stress state that far exceeds the yield strength of $\mathrm{Ag}$ and ordinary dislocation plasticity at lower stresses [31]. The influence of these two stress states is also evident in scanning electron microscope (SEM) images (Fig.2b-c) of the samples that show severe plastic flow at the bottom impacted region and discrete surface slip steps at the less deformed top region of the sample, which are characteristic of the ordinary plastic deformation governed by crystallographic slips of $\{111\}$ close-packed planes. Since the kinetic energy of impact $(2.3 \mathrm{~nJ})$ is significantly lower than the energy required to melt the Ag cube $(9.65 \mathrm{~nJ})$, no bulk melting is expected during the high-strain-rate deformation and the entire deformation process must occur in solid state (SI Sec.1). Creating a GNG structure in metals has been proposed [31-34] as a pathway to achieving both ultra-strength and -toughness that are usually mutually exclusive mechanical properties.

\subsubsection{Post-impact electron microscopy}

We performed high-resolution SEM analysis of the impacted samples and the preparation of thin TEM-lamella in an FEI Helios NanoLab 660 DualBeam system. We also prepared thin lamella in the Helios NanoLab system for subsequent S/TEM study. Prior to focused ion beam (FIB) 
milling, we coated the samples first with a layer of electron-beam deposited Pt and then a thick layer of ion-beam deposited $\mathrm{Pt}$ in order to protect the sample from direct $\mathrm{Ga}^{+}$ion damage. Then, we cut trenches and performed in-situ lift-out and attached the sample to a TEM half-grid. Finally, we thinned down the sample to below $100 \mathrm{~nm}$ using high energy (30keV) FIB milling, and subsequently polished under very low-energy ion beam $(2 \mathrm{keV})$ to minimize the damage caused by $30 \mathrm{keV}$ ions. The TEM specimens are analyzed in an FEI Titan Themis ${ }^{3}$ Scanning/Transmission Electron Microscope (S/TEM) operated at $300 \mathrm{keV}$ immediately after final polishing. We performed selective area diffraction (SAD) $(\sim 200 \mathrm{~nm}$ diameter probing area) to investigate the deformed nano/microstructure.

\subsubsection{Transmission Kikuchi diffraction analysis}

TKD was performed at the Center for Electron Nanoscopy of the Technical University of Denmark in an FEI Nova NANOSEM $600^{\mathrm{TM}}$ equipped with a field-emission gun and an $\mathrm{e}^{-}$Flash HR camera ( $\mathrm{GmbH}$, Berlin, Germany). In order to prevent oxidation and deterioration of the $\mathrm{Ag}$ microcubes during the transport from the United States to Denmark, they were coated with initially an e-beam deposited $\mathrm{Pt}(\sim 500 \mathrm{~nm})$ and then i-beam deposited $\mathrm{Pt}(\sim 3 \mu \mathrm{m})$. This was carried out in the FEI Helios NanoLab 660 DualBeam system. Then, we cut trenches and performed bulk in-situ lift-out and attached the sample to a TEM half-grid before shipping (Pt-coated sample width 10 $\mu \mathrm{m})$. The sample was thinned in Denmark using FIB to achieve electron transparent lamella. An accelerating voltage of $30 \mathrm{kV}$ for $\mathrm{Ga}^{+}$was used for the penultimate step. For the final polishing step that removes any amorphous region created due to i-beam damage, $2 \mathrm{kV} \mathrm{Ga}^{+}$with a current of $6 \mathrm{pA}$ was used to expose the thin sample at an incident angle of $7^{\circ}$ on both sides for $10 \mathrm{~min}$.

The TKD measurement was performed on the prepared electron transparent lamella in a square grid with step size of $4 \mathrm{~nm}$. An electron probe current of $1.7 \mathrm{nA}$ at an acceleration voltage of $30 \mathrm{kV}$, and camera exposure time of $8 \mathrm{~ms}$ was utilized. The TKD measurement was carried out in an on-axis setup [35]. The ESPRIT 2 Software from Bruker was used for the analysis of the acquired TKD data. A conservative approach for data cleaning was employed which was limited to just removal of wild points.

\subsection{Molecular dynamics simulations}

To understand the dynamic formation of the hcp phase in Ag, we performed molecular dynamics (MD) simulations of Ag nanocubes. Due to the computational limitations of the MD simulations, we simulate the impacts of $(65.5 \mathrm{~nm})^{3} \mathrm{Ag}$ nanocubes $\left(\sim 160^{3} \mathrm{fcc}\right.$ unit cells with lattice 
constant $\sim 0.409 \mathrm{~nm}$ at $0 \mathrm{~K} ; 16,384,000$ atoms) to model the experiments on Ag microcubes. All simulations are performed with the LAMMPS code [36,37] and the material $(\mathrm{Ag})$ is modeled with the Embedded atom method interatomic potential developed by Williams et al. [38].

The initial temperature of the samples is set to a constant value of $300 \mathrm{~K}$. The initial configuration is relaxed by integrating the equations of motion for 30 ps without applying any load. During this relaxation process the crystal is allowed to expand and/or contract independently in each orthogonal direction allowing to minimize the energy and the pressure of the nanocube using NPT ensemble with a Nosé-Hoover thermostat and a time-step of $5 \mathrm{fs}$.

Once the sample is equilibrated to $300 \mathrm{~K}$, and the pressure minimized to zero, an initial translational velocity is applied to the atoms to generate the impact velocity. Then, the positions of the atoms are integrated under the NVE ensemble for about 550 ps. The NVE ensemble ensures that no extra energy is added to the computational cell. The rigid wall was generated using a command which includes an atomic interaction at specific location $(x=0)$. The interaction is given by a Lennard-Jones-type potential, i.e.,

$$
E_{W}(r)=\epsilon\left(\frac{2}{15}\left(\frac{\sigma}{r}\right)^{9}-\left(\frac{\sigma}{r}\right)^{3}\right) ; \quad r<r_{C}
$$

where $r$ is the minimum distance between the particle and the wall, $\epsilon=1 \mathrm{eV}, \sigma=0.1 \mathrm{~nm}$, and $r_{c}=0.25 \mathrm{~nm}$ is a cut-off radius. Other choices of the wall generate the same results.

From the MD data we compute the average strain rate by using the change of the length of the sample and the time required to do that:

$$
\dot{\epsilon}=\frac{\epsilon}{\Delta \mathrm{t}}=\frac{\Delta l}{l_{0}} \frac{1}{\Delta t}=\frac{1}{\Delta t}\left(\frac{l-l_{0}}{l_{0}}\right) \text {, }
$$

where $l$ is the final length, $l_{0}$ is the initial length, and $\Delta t$ is the elapsed time at which $l$ and $l_{0}$ have been measured. For the [100]-face impact, we estimated the strain rate to be $\sim 2.4 \times 10^{9} \mathrm{~s}^{-1}$, while the strain rate for the [110]-edge impact was $\sim 3.7 \times 10^{9} \mathrm{~s}^{-1}$. These average values of the strain rate are less than an order of magnitude larger than that estimated from experiments $\left(\sim 8 \times 10^{8} \mathrm{~s}^{-1}\right)$ and is mainly due to the size mismatch of the cube. Additionally, we should mention that the instantaneous strain at each section of the sample could change, especially for the [110]-edge impact since the cross-section is strongly increasing as time goes by, making the deformation and strain non-homogenous. The inherent limitation of the number of atoms in MD makes it very challenging to match the experimental sizes. Indeed, with more than 16 million atoms, the simulations represent approximately the limit that one can achieve with the state-of-the-art MD techniques. Other coarse- 
grained techniques, such as the quasi-continuum method, cannot be applied since atomic resolution is required everywhere in the sample because the deformation is not localized near the impact area. With an overall strain-rate mismatch ratio of $\sim 5$ between simulations and experiments, we do not expect big differences. Other limitations, such as the validity of the potential under extreme pressures might play a more important role.

The strength of the simulation cell is quantified using virial stresses [37] that are computed from,

$$
\sigma_{\alpha \beta}=-\frac{1}{V}\left(\sum_{i} \frac{p_{\alpha}^{i} p_{\beta}^{j}}{m}+\sum_{i} \sum_{j} r_{\alpha}^{i j} f_{\beta}^{i j}\right) ; \quad \alpha, \beta=x, y, z
$$

where $\sigma_{\alpha \beta}$ are the components of the stress tensor, $V$ is the total volume of the simulation cell, $p_{\alpha}^{i}$ is the component $\alpha$ of the linear momentum $\left(p_{\alpha}^{i}=m v_{\alpha}^{i}\right)$ of atom $i, r_{\alpha}^{i j}=x_{\alpha}^{i}-x_{\alpha}^{j}$ is the component $\alpha$ of the relative distance vector between atoms $i$ and $j$, and $f_{\beta}^{i j}$ is the component $\beta$ of the force vector on atom $i$ due to atom $j$. Since the sample is initially traveling with a large translation speed, we subtracted their center of mass velocity and use the components of linear momentum without the velocity of the center of mass.

We use two independent methods to quantify the amount of plastic work generated in the simulation cell. The first quantity is the von Mises equivalent stress given by,

$$
\sigma_{e}=\left(\frac{1}{2} \sum_{\alpha>\beta}\left(\sigma_{\alpha \alpha}-\sigma_{\beta \beta}\right)^{2}+3 \sum_{\alpha>\beta} \sigma_{\alpha \beta}{ }^{2}\right)^{\frac{1}{2}}
$$

The von Mises equivalent stress allows introducing the stress triaxiality factor, which is a direct measure of the plastic deformation in the sample. The stress triaxiality factor is given by,

$$
\xi=\frac{\sigma_{m}}{\sigma_{e}}
$$

where $\sigma_{m}=\frac{1}{3}\left(\sigma_{11}+\sigma_{22}+\sigma_{33}\right)$ is the hydrostatic stress.

In order to provide a more comprehensive study of the impact, we calculated the spatialtemporal evolution of stress and temperature, again using the kinetic energy of the atoms and virial stress. The procedure was reproduced from a laser irradiation simulation where the pressure and temperature were described as a function of the space-time [39]. In order to study the distribution of temperature and stresses, we divided the computational cell into bins of equal size along the $\mathrm{x}$ direction (normal to the impact plane). For all simulations, we took $N_{b i n s}=40$. Then, for each bin, we computed the components of the virial stress tensor, and the temperature using the local kinetic 
energy. Then, we subtracted the temperature of the center of motion. In order to compute the virial stress, we needed to take a reference volume. Here, we used for each bin a volume given by

$V_{\text {bin }}=\frac{V_{c u b e}}{N_{\text {bins }}}$ to compute the virial stress. These quantities were processed on the fly, and dumped to a file every 1 ps. With the temperature and stress for different times and locations, we generated the contour plots shown in Figures 8 and 9.

\section{Identification of hep phase}

\subsection{Experiment}

\subsection{1. [100] impact}

The thin lamella, (Fig.3a) obtained from an Ag microcube that was impacted along [100] direction (face-on impact) and examined in TEM within 24 hours from impact, exhibits regions with SAD patterns that correspond to an hcp crystal structure (Fig.3b-d). Each diffraction pattern was examined with several different zone axes of the $2 \mathrm{H}$ (Fig.3g) and $4 \mathrm{H}$ (Fig.3h) polytypes to find the best fit. We performed detailed error analysis to determine the most probable polytype and zone axis for each pattern (SI Tables 6-8). Overall, 4H was consistently a better fit for all SAD patterns.

Since the hcp grain orientations were not primarily in low-order zone axes, we additionally performed diffraction peak analysis and compared our data to diffraction peak data of various $\mathrm{Ag}$ polytypes from the American Mineralogist Crystal Structure Database. The benefit of this method is that it does not rely on the determination of a zone axis or an indexed pattern from the software and the data is extracted directly from the diffraction pattern. The intensity curve for each diffraction pattern is calculated by integrating the intensities over $\theta=0$ to $2 \pi$ for each distance $d$ from the zone axis spot: $I(d)=\int_{0}^{2 \pi} I(d, \theta) d \theta$. Intensities are normalized by the maximum intensity and then the data from all diffraction patterns are averaged together to create the intensity curve for one sample. Figure 3f shows diffraction data compiled from two different samples with intensity peaks for fcc and 4H Ag-polytypes overlaid. Sample-1 and Sample-2 intensity plots are averages of 21 and 51 SAD patterns obtained at various locations on the TEM lamellae, respectively. Each sample has a different range of d-spacings, likely due to different diffraction conditions. The peaks of Sample-2 are much more distinct due to consistency between individual diffraction patterns. Sample-1, on the other hand, has very broad peaks since the individual diffraction patterns have varied peak positions and intensities. This can be attributed to a larger degree of micro-strain or various different polytypes being present in the sample. Several peaks in each sample correspond to $4 \mathrm{H}$ peaks, while 
only one peak in Sample-1 at around $8.12 \mathrm{~nm}^{-1}$ shows closer agreement with 3C. This suggests most of the sample has transformed to $4 \mathrm{H}$ while a small amount of fcc phase may still be present. Differences in our diffraction data compared to the intensity peaks from the database are potentially due to the different diffraction method used; data from the American Mineralogist Crystal Structure Database is obtained via x-ray diffraction while our data was obtained using TEM diffraction for which artifacts such as double diffraction are possible.

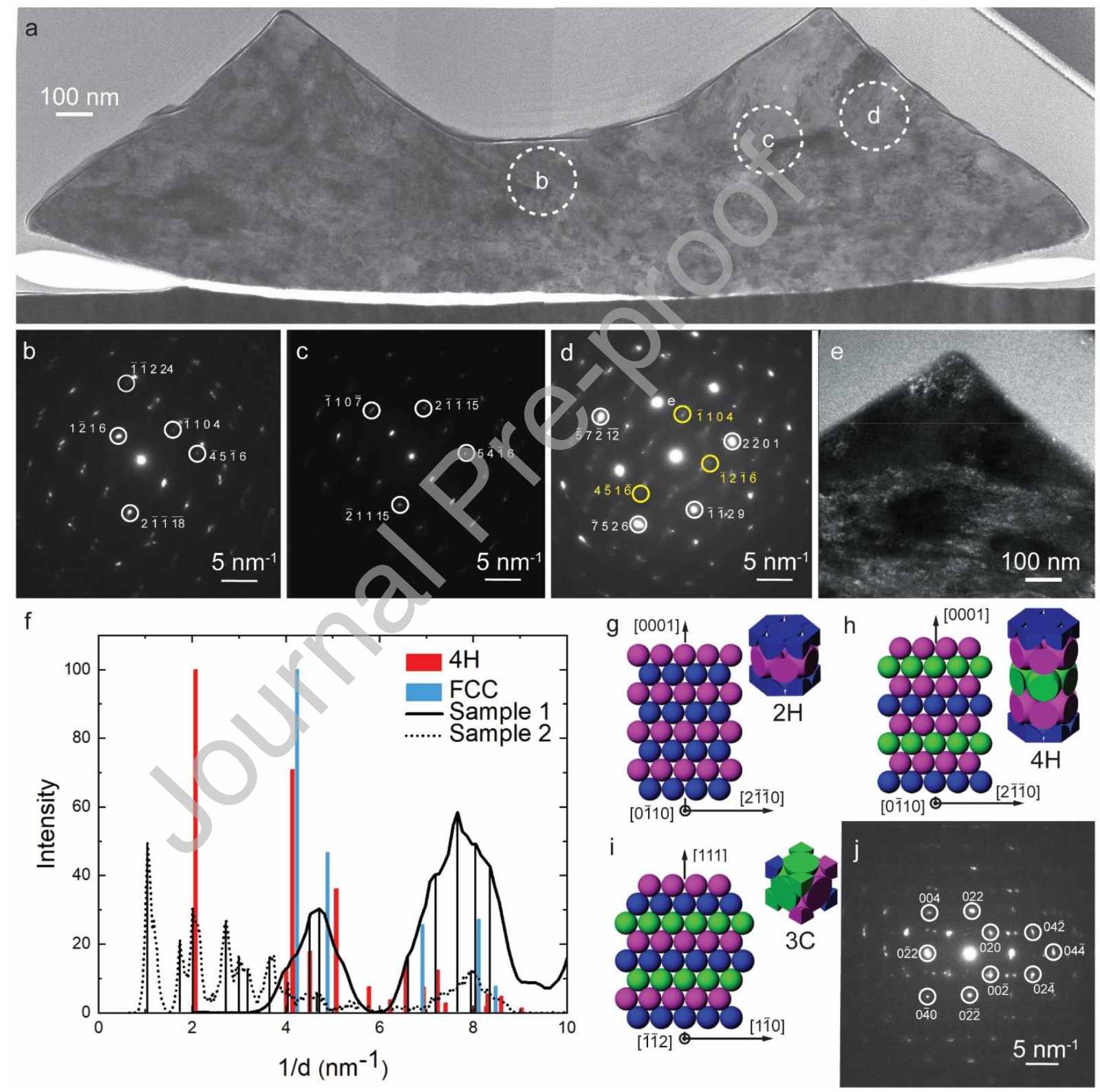

Figure 3. Martensitic phase transformation in Ag microcubes impacted along the [100] direction: (a) TEM image of a thin lamella obtained from the middle section of the impacted sample within 24 hours from impact, (b-d) SAD patterns seen at the location indicated as 'b' (hcp zone axis: [10 $\overline{2} \overline{8} 3]$ ), 'c' (hcp zone axis: [ $\overline{2} 19 \overline{17} 3])$, and 'd' (hcp 
grain 1 (indicated in white) zone axis: [ $\left.\begin{array}{llll}1 & 3 & \overline{4} & 4\end{array}\right]$, hcp grain 2 (indicated in yellow) zone axis: [ [ $\left.10 \overline{2} \overline{8} 3\right]$ ) in $\mathbf{d}$ showing diffraction from hcp grains; diffraction from two of the multiple adjacent hcp grains are indexed in white and yellow in d, (e) dark field TEM image corresponding to [ [ $\left.\begin{array}{llll}\overline{1} & 1 & 0 & 4\end{array}\right]$ diffraction spot indicated as ' $\mathrm{e}$ ' in $\mathbf{d}$, showing hcp grains as small as $10 \mathrm{~nm}$, (f) intensity peak curves of compiled diffraction data from two samples with $4 \mathrm{H}$ and FCC peaks from the database overlaid, (g-i) illustrations of the close-packed planes of $\mathrm{Ag}$ in $2 \mathrm{H}(\mathrm{g}), 4 \mathrm{H}(\mathrm{h})$, and fcc (i) phases; insets show unit cells of respective phases (blue, purple, and green colors indicate the atoms that belong to A, B, and C stacking planes, respectively). (j) SAD pattern obtained after further ion-beam thinning showing the characteristics of reverted fcc grains.

Our detailed analysis reveals the presence of an hcp phase in [100] impacted samples, most likely of a $4 \mathrm{H}$ structure containing $\mathrm{ABCB}$ stacking sequence. This suggests that a martensitic dynamic phase transformation must have occurred due to impact, transforming the fcc crystal structure to an hcp structure in the nanosecond timescale. Upon further thinning of the same sample to less than $50 \mathrm{~nm}$ using FIB, we observe that the SAD patterns (Fig.3j) now represent the characteristics of an fcc structure. This suggests a secondary phase transformation that transforms the $4 \mathrm{H}$ phase back to an fcc (3C) structure can occur due to stress-relaxation [40] in a thinned sample that has undergone significant exposure to ion beam and electron beam radiation [27].

\subsection{2. [110] impact}

We additionally investigated Ag microcubes that were impacted along the [110] direction (edge-on impact) (Fig.4). The post-impact SEM image of the sample (inset of Fig.4a) shows severe plastic deformation at the bottom impacted region and surface slip steps from the characteristic crystallographic slips at the less-deformed top region of the sample. Though the nature of the exterior deformation features is similar to the [100] impacted samples, the plastic flow is confined to much narrower bottom impacted region of the sample. Interestingly, the thin lamella obtained from the middle section of the [110] impacted sample (Fig.4a) and examined similarly in TEM within 24 hrs from impact does not show the presence of hcp phase. The sample consists of nanocrystalline fcc regions at the bottom impacted side of the sample and large grains of crystalline fcc regions towards the top and middle sections of the sample (Fig.4c-f). This further confirms that the severe plasticity has been confined to the bottom impacted region due to the wave interactions with adjacent free surfaces of the sample that form a wedge-shape facing the impact direction. The SAD patterns obtained at the bottom corners of the sample show significant amorphous regions suggesting that rapid melting and quenching of the Ag cubes must have occurred during the [110] impact (Fig.4f). Measurement of the angles of rotation, relative to the grain orientation at the center 
(indicated as ' $\mathrm{O}$ ' in Fig.4a), in the SAD patterns obtained horizontally on this sample shows grain rotations consistent with the plastic deformation in the sample that flows outward as the $\mathrm{Ag}$ microcube dynamically deforms (Fig.4b).

a
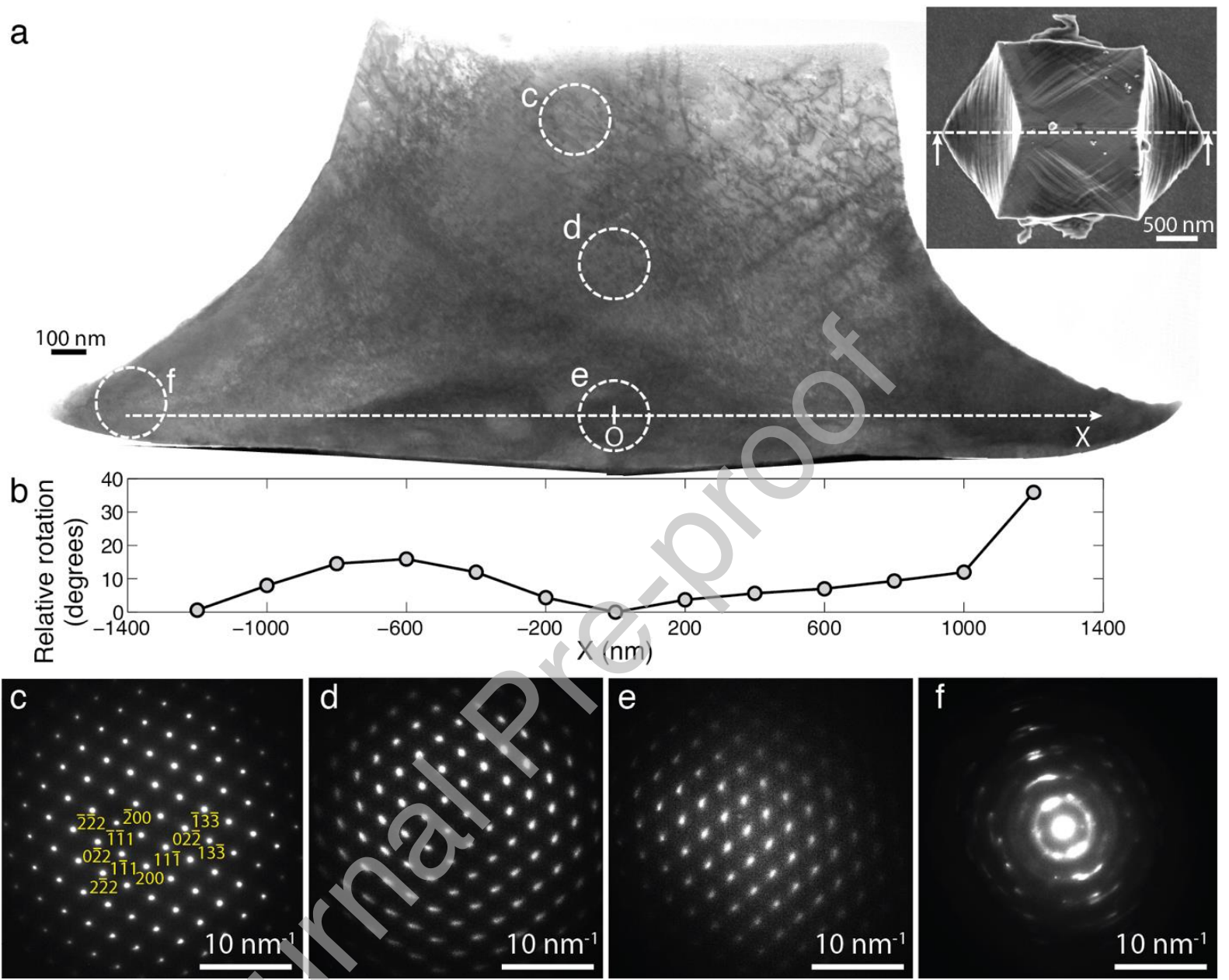

Figure 4. Impact-induced deformation structure of the Ag microcube impacted along [110] direction: (a) TEM image of a thin lamella obtained from the middle section of the impacted sample within 24 hours from impact; inset shows a top SEM view of the sample, (b) relative grain rotations (with respect to the pattern at origin ' $O$ ') measured from SAD patterns obtained along the horizontal X-axis at $200 \mathrm{~nm}$ intervals, (c-f) SAD patterns obtained at various locations as indicated in ' $\mathbf{a}$ ' showing single crystalline pattern at the top and middle regions and highly nanocrystalline pattern and amorphous Ag (diffused rings) at the bottom edge regions.

\subsection{Molecular dynamics (MD) Simulations}

\subsection{1. [100] impact}

The molecular dynamics simulations allow us to track the temporal evolution of the atomic structure (Fig.5a). We investigate the deformation nano- and atomic-structures of the sample using 
the local atomic structure calculated by the common neighbor analysis (CNA) algorithm implemented in OVITO [41]. Initially (0 ps), $97 \%$ of atoms are in the fcc crystal structure while $\sim 3 \%$ are found to be of other crystal symmetries, which primarily includes the surface atoms all around the nanocube. After the impact, the local structure significantly changes as the function of time. At 4 ps, $92 \%$ of the atoms are in fcc structure while $1.4 \%$ are in body-centered-cubic (bcc) structure and $5.6 \%$ are in other crystal structures. This change is due to the shock wave propagation in the sample that generates lattice distortion and eventually results in plastic deformation. At $34 \mathrm{ps,}$ the sample contains only $62.5 \%$ of fcc atoms while $19.4 \%$ of the atoms have transformed into a new hcp phase (bcc $\sim 2.6 \%$, other $\sim 14.7 \%$ ). The hcp phase forms due to high emission of partial dislocations that form stacking faults (see Sec. 4.1 for more details). Large regions of hcp phase form in the sample as the deformation progresses further (hcp regions are shown in red in Fig.5b). Closer examination of the large hcp regions that have formed due to impact (Fig.5b inset) shows stacking sequence of $2 \mathrm{H}$ polytype (stacking sequence $\mathrm{AB}$, lattice constants $\mathrm{a}=0.283 \mathrm{~nm}$ and $\mathrm{c}=$ $0.638 \mathrm{~nm}$ ) rather than $4 \mathrm{H}$ polytype (stacking sequence $\mathrm{ABCB}$, lattice constants $\mathrm{a}=0.288 \mathrm{~nm}, \mathrm{c}=$ $1.0 \mathrm{~nm}$ ) as found from the experimental SAD patterns. After $51 \mathrm{ps}$, the percentage of the hop phase reaches a steady state. However, other types of atoms dynamically recrystallize into an fcc structure. A steady state is reached after $\sim 200$ ps, when the temperature and kinetic energy stabilize (Fig.S2(ab) in SI). The final sample contains $69.9 \%$ fcc phase, $14.0 \%$ hcp phase, $0.8 \%$ bcc phase, and $15.2 \%$ in other phases. The time evolution of the deformation nano- and atomic-structures of the $\mathrm{Ag}$ nanocube impacted along [100] crystal symmetry direction is shown in the supplementary Video S1 and snapshots are presented in Figure 5c. 

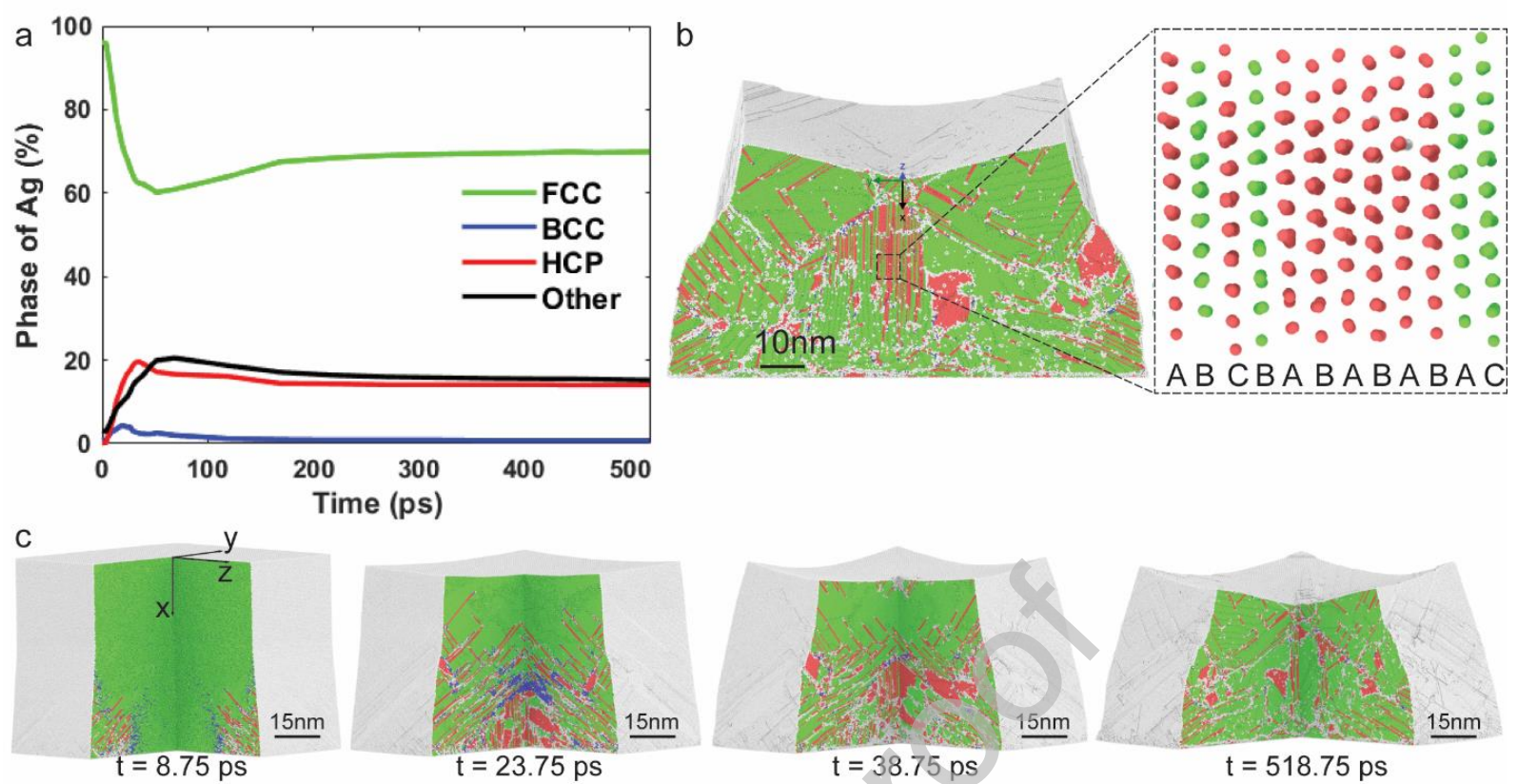

Figure 5: Phase composition of [100] impacted cube found in MD simulations: (a) relative amount of various phases present as a function of time in the [100] impacted Ag nanocube, (b) (001) mid-section of the sample showing large regions with the new hcp $(2 \mathrm{H})$ phase; inset shows the magnified view of the stacking sequence where the red and green indicate atoms in hep and fcc phases, (c) snapshots of the phase content evolution of the [100] impacted Ag nanocube over time.

\subsection{2. [110] impact}

We also examined samples impacted along the [110] direction using MD simulations (Fig.6). As a result of the large initial velocity given to the sample, and because the sample is hitting the wall on its edge without much of lateral geometrical constraints, there is melting of the sample that is evident in the simulation through the evolution of the atoms in an amorphous structure (labelled as "other" in Fig.6a). Steady state is reached approximately 175 ps after impact, when the temperature and kinetic energy stabilize (Fig.S2(c-d) in SI). The portion of atoms that become amorphous is $\sim 18 \%$. We also notice that a large $76 \%$ portion of the atoms remain in fcc structure at the end of the simulation (Fig.6b). Finally, we see that only $8 \%$ of the atoms are in a local hcp structure. However, this structure corresponds to several individual stacking fault defects (Fig.6b inset) emitted between Shockley partial dislocations and does not reflect a large hop phased domain as in the [100] impacted sample. The time evolution of the deformation nano- and atomic-structures of the Ag nanocube impacted along [110] crystal symmetry direction is shown in the supplementary Video S2 and snapshots are presented in Figure 6c. 


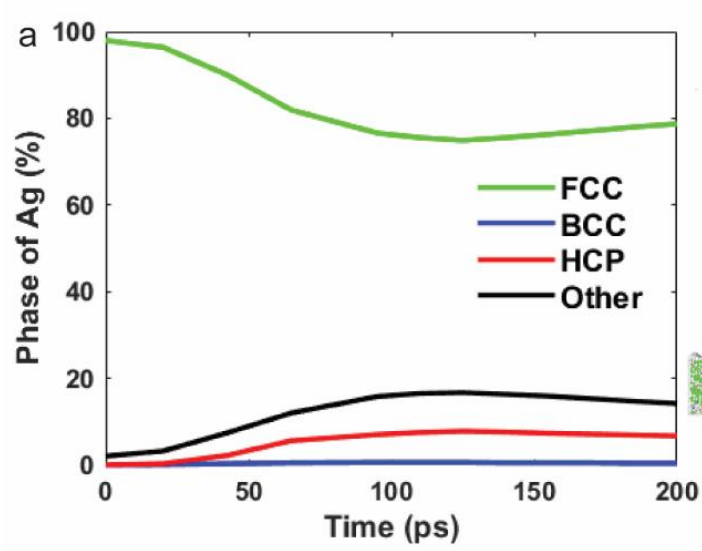

b
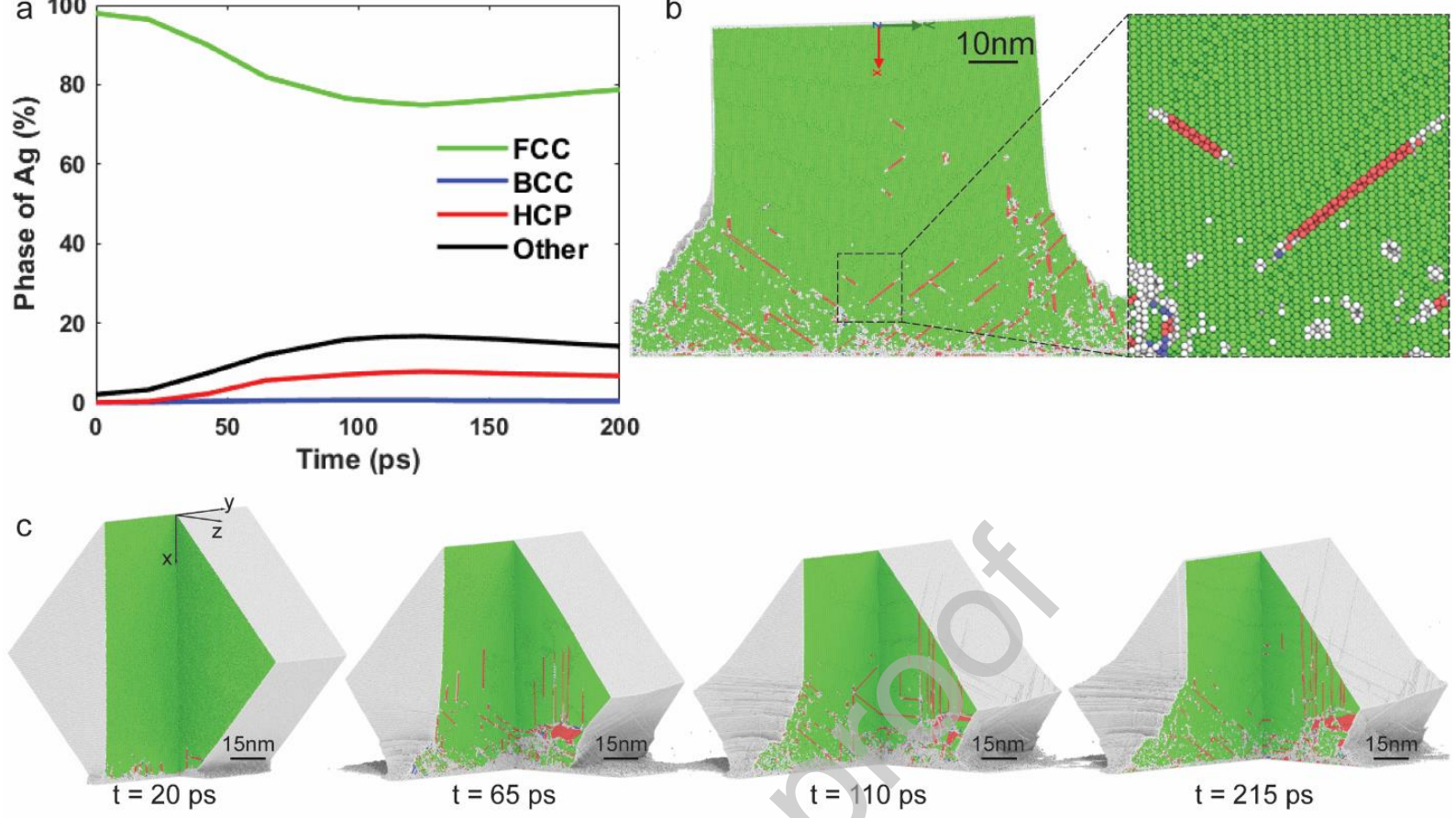

Figure 6: Phase composition of [110] impacted cube found in MD simulations: (a) relative amount of various phases present as a function of time for the [110] impacted Ag nanocube, (b) (001) mid-section of the sample with no hcp regions; inset shows the magnified view of small stacking faults, (c) snapshots of the phase content evolution of the [110] impacted Ag nanocube over time.

To verify the occurrence of melting in the [110] impacted cubes, we plot radial distribution functions (RDFs) for the [100] and [110] impacted cubes (Fig.7). The RDF describes how the density of a material varies as a function of the distance. In crystalline materials, it is useful to identify neighboring shells, which are denoted by peaks in the RDF. The RDF for the [100] impacted sample far from the impact surface shows the existence of multiple peaks at $\sim 0.3$ $\mathrm{nm}, \sim 0.42 \mathrm{~nm}$, and $\sim 0.52 \mathrm{~nm}$. These peaks correspond to the position of the first, second, and third neighboring shells in the fcc structure. Thus, even though the sample has recrystallized, it shows a crystalline structure that is consistent with the fcc lattice structure. For the [110]-edge impact (blue and black), the RDF was computed at two different locations. First, the RDF is computed in the middle of the sample. We see that the RDF for the [110]-edge impact simulation is similar to the [100]-face impact, showing that the sample is crystalline in the middle of the simulation cell. However, when the RDF is computed for the atoms near the surface (black), it shows an amorphous structure, characterized by the removal of the second peak and significant 
weakening and broadening of the third peak in the RDF. This is a clear indication that the structure is truly amorphous and in a liquid-like phase.

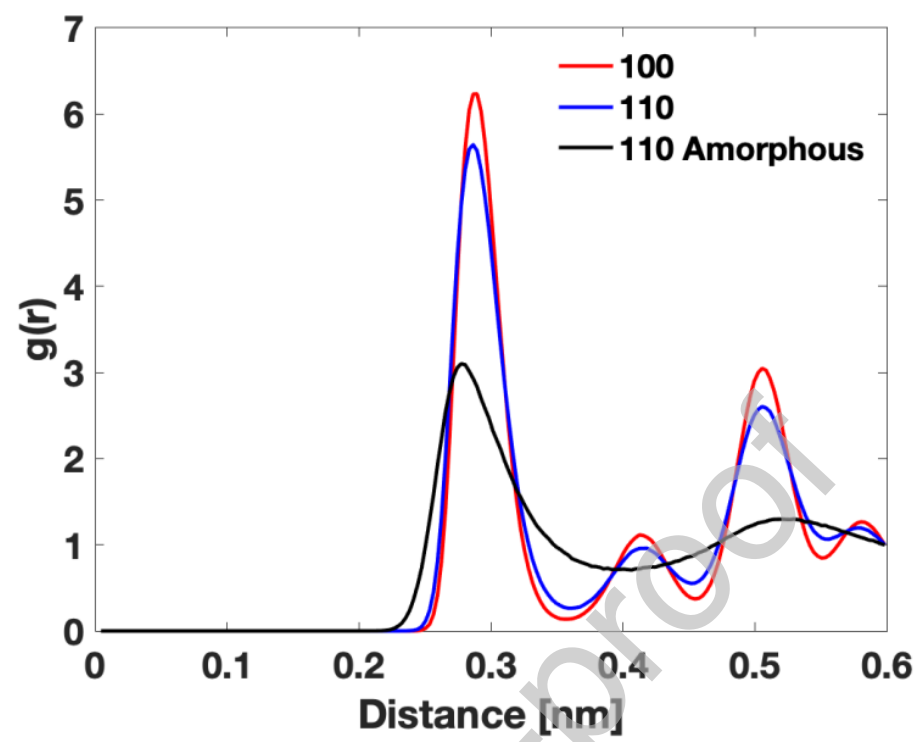

Figure 7: Radial distribution functions (RDFs) from MD simulations: RDF for the [100]-face impact simulation (red). The peaks indicate different shells of neighboring atoms in the fcc structure, RDF at the middle of the sample for the [110]-edge impact simulation (blue), RDF for atoms near the wall for the 110-edge impact simulation (black). The suppression of peaks indicates an amorphous structure.

\section{Creation and stability of hcp phase}

\subsection{Mechanisms driving hcp phase transformation}

To determine how the fcc to hcp phase transition occurs in [100] impacted samples, we examined the atomic-structure evolution and stress-time histories provided by the MD simulations (Fig.8). As the sample impacts the rigid target in the [100] orientation, an elastic shock wave develops and propagates through the sample. The oscillatory behaviour apparent in the hydrostatic stress curve (Fig.8a) is characteristic of shock wave propagation and interaction with the sample surface. The period of this fluctuation is between 26 to $32 \mathrm{ps,} \mathrm{which} \mathrm{is} \mathrm{very} \mathrm{close} \mathrm{to} \mathrm{the} \mathrm{time} \mathrm{the}$ elastic shock wave takes to travel twice the length of the $\sim 65.5 \mathrm{~nm}$ sized nanocube sample. On the other hand, the von Mises equivalent stress (Fig.8a) initially increases higher than $1.3 \mathrm{GPa}$ in a very short time ( $10 \mathrm{ps}$ ) and remains higher than $1 \mathrm{GPa}$ for around $\sim 80 \mathrm{ps}$. The von Mises equivalent stress then gradually reaches zero as the kinetic energy due to the impact velocity is converted to plastic work. The resultant stress triaxiality factor (Fig.8b) is very dynamic because of the 
oscillatory hydrostatic stress component. Both the hydrostatic and von Mises stresses approach zero by around $200 \mathrm{ps}$ when steady state is reached, and the microstructure remains approximately the same (Fig.8g; $\mathrm{t}=180 \mathrm{ps}$ ) until the end of the simulation. This indicates that the GNG nanostructure (Fig.8g) and the martensitic phase transformation happen in a sub-nanosecond timescale.

Using the procedure outlined in Section 2.2, we obtained the spatial distributions of temperature and normal stress as a function of time (Fig.8c-f). As expected, the stress has large fluctuations across the sample for different times (Fig.8c), indicating that a shock wave was propagated in the sample within the first 20 ps (Fig.8e). The slope of the space-time plot represents the wave speed, which was computed to be in the order of $\sim 3,680 \mathrm{~ms}^{-1}$ in good agreement with the speed of sound in $\mathrm{Ag}\left(3650 \mathrm{~ms}^{-1}\right)$. Behind the elastic precursor, the shock wave quickly increased the values of the stress above the yield strength of the material. For instance, at the impacted face the compressive stress is in the neighbourhood of $21 \mathrm{GPa}$. When the wave reached the end of the nanocube (indicated with a dashed line in the plots), the traction-free condition in the sample generated a change of sign in the stress, as can be seen in the figure at around the point $(60 \mathrm{~nm}, 18$ ps). The maximum recorded value of the tensile stress was about $\sim 3.8 \mathrm{GPa}$. Once the wave was reflected back, it reached the impacted end, and the process was reproduced again until the wave disappeared due to dissipation. The large shock wave propagated right after the impact carried a large amount of plasticity that triggered an avalanche of dislocations. 

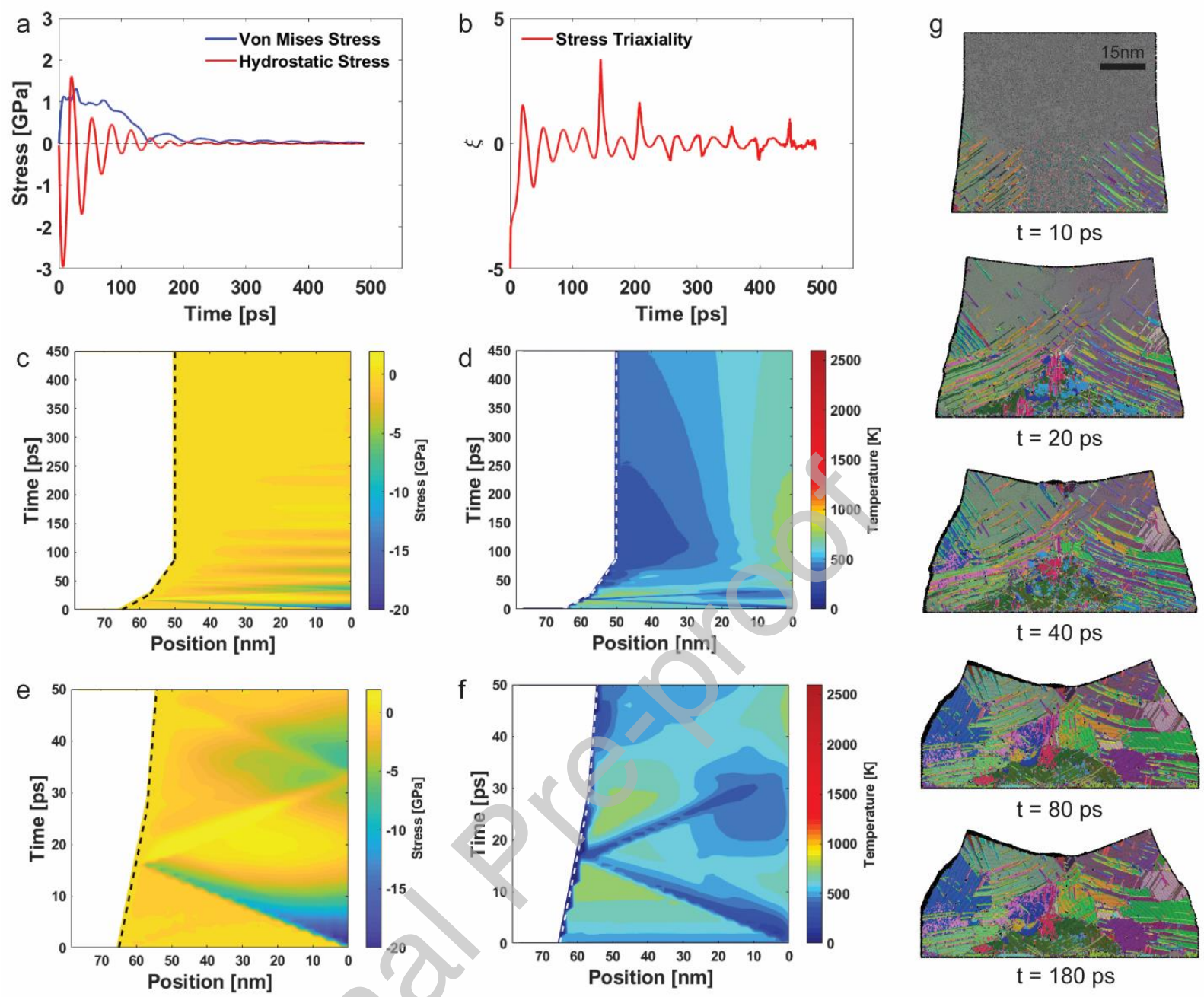

Figure 8. Structural evolution of [100] impacted nanocube: (a) the von Mises equivalent stress and the hydrostatic stress as a function of time, (b) the stress triaxiality factor as a function of time, (c) normal stresses as a function of position from the impact plane and time, (d) temperature as a function of position from the impact plane and time, (e) magnified space-time stress plot, (f) magnified space-time temperature plot, (g) microstrucural evolution of the [100] impacted Ag nanocube as a function of time.

Focusing on the temperature evolution, we observe that the initial impact quickly raised the temperature of the sample to about $750 \mathrm{~K}$ at the opposite end of the impacted face (Fig.8d,f). The shock wave also changes the temperature of the atoms immediately behind its front, indicating a complex thermo-mechanical coupling between temperature and plastic deformation. After the initial wave propagation, a large portion of the sample near the impacted face increased its temperature above $900 \mathrm{~K}$, however, this temperature gradient slowly reduced with time. For instance, at $450 \mathrm{ps,}$ the temperature was around $680 \mathrm{~K}$ at the impacted face while the opposite end was at $485 \mathrm{~K}$ 
(Fig.8d). This poor thermal conductivity is due to the generation of a gradient nano-grained structure, that is of the order of the mean free path of phonons in the sample, and thus, the newly generated grains make phonon scattering more often than in bulk. The unique temperature evolution of the nanocube impacted along the face indicates the generation of a rich nanostructure.

The shock wave which propagates between the sample's surfaces is the key driving force for creating stacking faults which lead to the martensitic phase transformation. Avalanches of partial dislocations are nucleated at the bottom lateral edges of the sample (Fig. $8 \mathrm{~g}$; $\mathrm{t}=10 \mathrm{ps}$ ) and propagate on $\{111\}$ planes along $\langle 110\rangle$ directions, forming large stacking faults. Shortly after impact, dislocations merge together in the mid-region of the sample leading to phase-transformation, formation and rotation of new grains, and dynamic recrystallization of the grains (Fig.8g; $t=20 \mathrm{ps}$ ). Once the compressive shock wave reaches the top free surface of the sample, it reflects back as a tensile wave with additional dislocations nucleated from the top free surface (Fig. $8 \mathrm{~g} ; \mathrm{t}=40 \mathrm{ps}$ ). During this period, the dislocations multiply in the bottom and middle impacted regions of the samples, leading to large hcp regions (indicated by red atoms in Fig.5c; t=38.75 ps). Severe outward plastic flow occurs at the bottom region of the sample with creation of new nanograins enclosed by thin amorphous regions (indicated by white atoms in Fig.5c; $\mathrm{t}=38.75$ ps). After 80 ps (Fig.8g; $\mathrm{t}=80$ ps) the microstructural changes slow and the microstructure remains approximately the same after 180 ps (Fig.8g; t=180 ps).

\subsection{Orientation effect on phase transformation}

Both the experimental results and simulations show that an hcp phase transformation does not occur in [110] impacted samples. We studied the differences between the [100] and [110] impact stress-state time histories provided by the MD simulation (Fig.8-9) to determine why a phase transformation did not occur in [110] impacted samples. A key distinction between the [110] and the [100] impact direction is that the hydrostatic stress is always negative during [110] impact (Fig.9a) with a minimum value of $\sim-0.35 \mathrm{GPa}$, a considerably smaller value than the one observed for the [100] impact direction (Fig.8a). This is already an indication that the hydrostatic stress state in the [110] impact is not as strong as in the [100] direction. Unlike the negative hydrostatic stress, the von Mises stress (Fig.9a) immediately increases upon impact, reaching values of $\sim 0.9 \mathrm{GPa}$. Such a large value is mainly achieved by the relatively large normal stress in the impact direction (first diagonal component of the stress tensor) as compared to the two other components of the stress tensor (Fig.S4 in SI). This is an indication that the stress is primarily 
uniaxial. The stress triaxiality shown in Figure $9 \mathrm{~b}$ also suggests that the large hydrostatic stress state does not develop in the [110] impacted sample. Initially, the stress triaxiality increases very quickly, and then decays and stabilizes after 10 ps. The initial peak is due to almost zero shear stresses for the first 50 ps (Fig.S4 in SI) which coincides with melting in a portion of the atoms in the simulation cell. The shear stresses build-up after 60 ps where several dislocations can be seen in the sample. After $10 \mathrm{ps,}$, while some fluctuations of the magnitude of the stress triaxiality are seen, the absolute values of the stress triaxiality are around \pm 0.6 , which is considerably smaller than in the [100] case (Fig.8b).

The spatial distributions of stress and temperature as a function of time reveal additional differences between the [100] and [110] impacted cubes. The space-time stress plot (Fig.9c) reveals that the impact generates a wave which propagates through the sample-similarly to the [100] impact orientation case. However, several differences appear with respect to the face impact. First, the wave speed is smaller, in accordance with the elastic modulus along the [110]direction. We also notice that the wave is much smoother than in the previous case, as can be seen from the range of colors behind the wave front (Fig.9e). For instance, at $10 \mathrm{ps}$ the compressive stress near the wall is around $\sim 2.1 \mathrm{GPa}$. While this is enough to nucleate dislocations, the stress values are considerably smaller than in the previous case. As a result, only a few dislocations are emitted, and most of the energy is used to melt the impacted area.

The presence of melting can be confirmed by examining the space-time temperature profile (Fig.9d,f). Regions near the impact plane reach temperatures over $2600 \mathrm{~K}$, which is well above the melting point of $\mathrm{Ag}(\sim 1230 \mathrm{~K})$. The heat is mainly concentrated near the impacted area while the rest of the sample remains at a much lower temperature. More importantly, we did not observe a change of the temperature behind the wave front, an indication that the energy carried by the wave was much smaller in this case. 

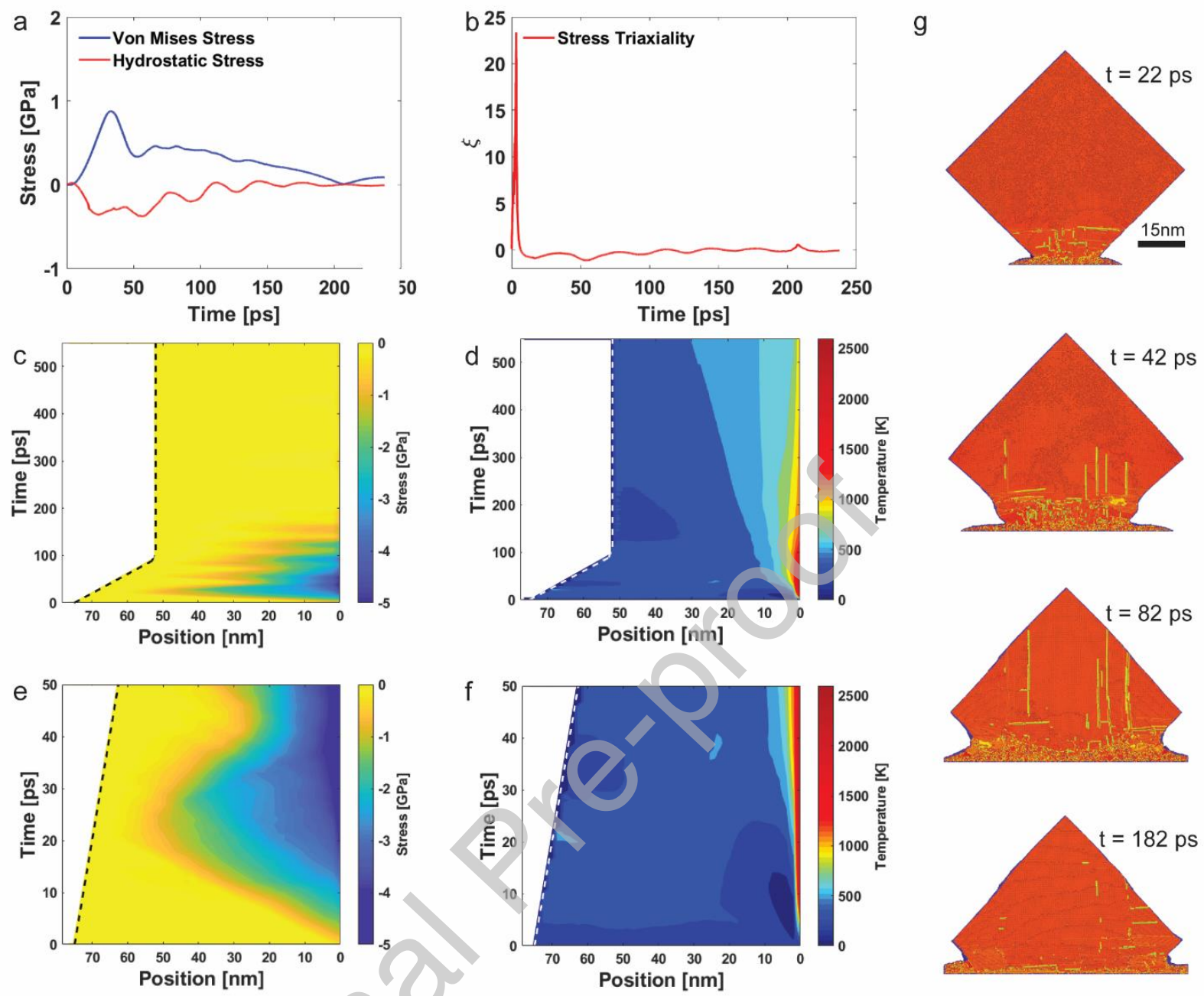

Figure 9. Structural evolution of [110] impacted nanocube: (a) the von Mises equivalent stress and the hydrostatic stress as a function of time, (b) the stress triaxiality factor as a function of time, (c) normal stresses as a function of position from the impact plane and time, (d) temperature as a function of position from the impact plane and time, (e) magnified space-time stress plot, (f) magnified space-time temperature plot, (g) microstrucural evolution of the [110] impacted Ag nanocube as a function of time.

Since the stress state of the [110] impacted sample is much milder compared to that of in the [100] impacted sample, a sustained shock and a hydrodynamic stress state does not arise in the sample - which is necessary for hcp phase transformation as discussed in Section 4.1. A gradientnano-grained structure is not formed as in the [100] impacted sample (Fig.8g), but instead, amorphous regions near the impacted edge are formed through melting (Fig.9g). This is also reflected in our experimental observations where the newly formed nanograins are confined to a narrow bottom band of the sample while the top and middle regions remain nearly single- 
crystalline. Additionally, the phase transformation may not have been favourable in [110] impact, because only a few slip systems are simultaneously active for a [110] loading direction.

Recent MD simulations of impacting Ag nanocubes[42] and nanoparticles [43] also have predicted the potential formation of small hcp domains during impact, though their prominence and mechanisms responsible for such phase transformation remained unclear due to the extremely small simulation cells (only up to $\sim 12 \mathrm{~nm}^{3}$ size). Our experimental observations on Ag microcubes and the MD simulations of larger $\left(\sim(65 \mathrm{~nm})^{3}\right) \mathrm{Ag}$ nanocubes clearly show that the unique fcc-to-hcp martensitic phase transformation and the nature of GNG structure formation are highly dependent on the intrinsic crystal symmetry and the particle shape symmetry along the impact direction.

\subsection{Relative stability of $2 \mathrm{H}, 4 \mathrm{H}$, and $3 \mathrm{C}$ phases}

MD simulations of [100] impacted samples show transition to the $2 \mathrm{H}$ phase (Fig.5b). However, since the experimental SAD observations consistently show close resemblance to the $4 \mathrm{H}$ phase, the metastable $2 \mathrm{H}$ phase that formed due to impact must have transformed back to the comparatively stable $4 \mathrm{H}$ phase either spontaneously or during the duration of the sample preparation. The necessary translation of only one [111] plane out of every four adjacently-stacked planes to cause a phase transformation from $2 \mathrm{H}$ to $4 \mathrm{H}$ suggests that the elastic strain energy stored in the deformed microcube may have caused a spontaneous phase transformation from $2 \mathrm{H}$ to $4 \mathrm{H}$ phase during the time from impact to the TEM analysis, i.e. 24 hrs (see SI Sec.2 for geometric analyses of the transformation among different polytypes of $\mathrm{Ag}$ ). The high concentration of vacancies that is generated due to impact may also have contributed to such secondary transformation of the metastable $2 \mathrm{H}$ phase (see SI Sec.5 for estimation of vacancies). More interestingly, unlike in the MD model, the entire mid-cross-section of the sample examined in TEM shows only hep grains rather than a mixture of fcc and hep phases. An inherent limitation of the MD model is that the fcc interatomic potential is not energetically favourable to predict the hcp phase formation (SI Sec.6). Regardless of this limitation, the large domains of hcp phase found in MD model are an indicator of the fcc structure's strong preference to transform into the hcp phase as observed in experiments where nearly the entire sample transforms into the hcp phase.

\subsection{Recrystallization involves transformation back to fcc phase}

To understand the stability of the unique hcp phase of Ag over time, we examined a thin lamella prepared from the middle (100) section of a sample impacted along the [100] direction 
approximately 7 days after impact (Fig.10a). We used the transmission Kikuchi diffraction (TKD) technique[35] to obtain the structure and orientation of individual grains across the entire crosssection (Fig.10b). The initial extremely small nanograins have coalesced and grown into slightly larger nanograins over the week. The sample shows a GNG structure containing fcc crystals oriented primarily at their [211] as well as [221] and [110] directions parallel to the impact direction (Fig.10b). Such orientation changes from the initial [100] orientation cannot be explained by ordinary crystal plasticity alone. Most interestingly, almost no grains are found at the initial impact orientation of the cube $\sim 100]$ revealing the complex nature of the dynamic deformation consisting of the GNG formation due to the interplay between different stress-states and the fcc-to-hcp martensitic phase transformation, and the subsequent continuous recrystallization processes. The coordinated shift of high number (eight) of different [111] planes necessary for a $4 \mathrm{H}$ to $3 \mathrm{C}$ transformation (SI Sec.2) suggests that this direct reverse-transformation process is unlikely to occur spontaneously without additional energy or a longer period of continuous (static) recrystallization at room temperature. Our previous study[31] on impacted Ag microcubes has shown that a continuous recrystallization process occurs at room temperature over days and weeks without any external thermal annealing, primarily driven by the strain energy stored in the material from the high-strain-rate deformation. The $4 \mathrm{H}$ to $3 \mathrm{C}$ transformation has also been seen in thermally annealed Ag nanoparticles[19] and severe electron-beam-irradiated Ag nanowires[23]. The absence of hcp grains in the sample investigated by TKD analysis suggests that the hcp phase is metastable in the impacted samples and the strain energy stored in the material and the high concentration of vacancies[44] generated (SI Sec.5) during dynamic deformation results in a transformation back to the fcc phase through continuous recrystallization. 

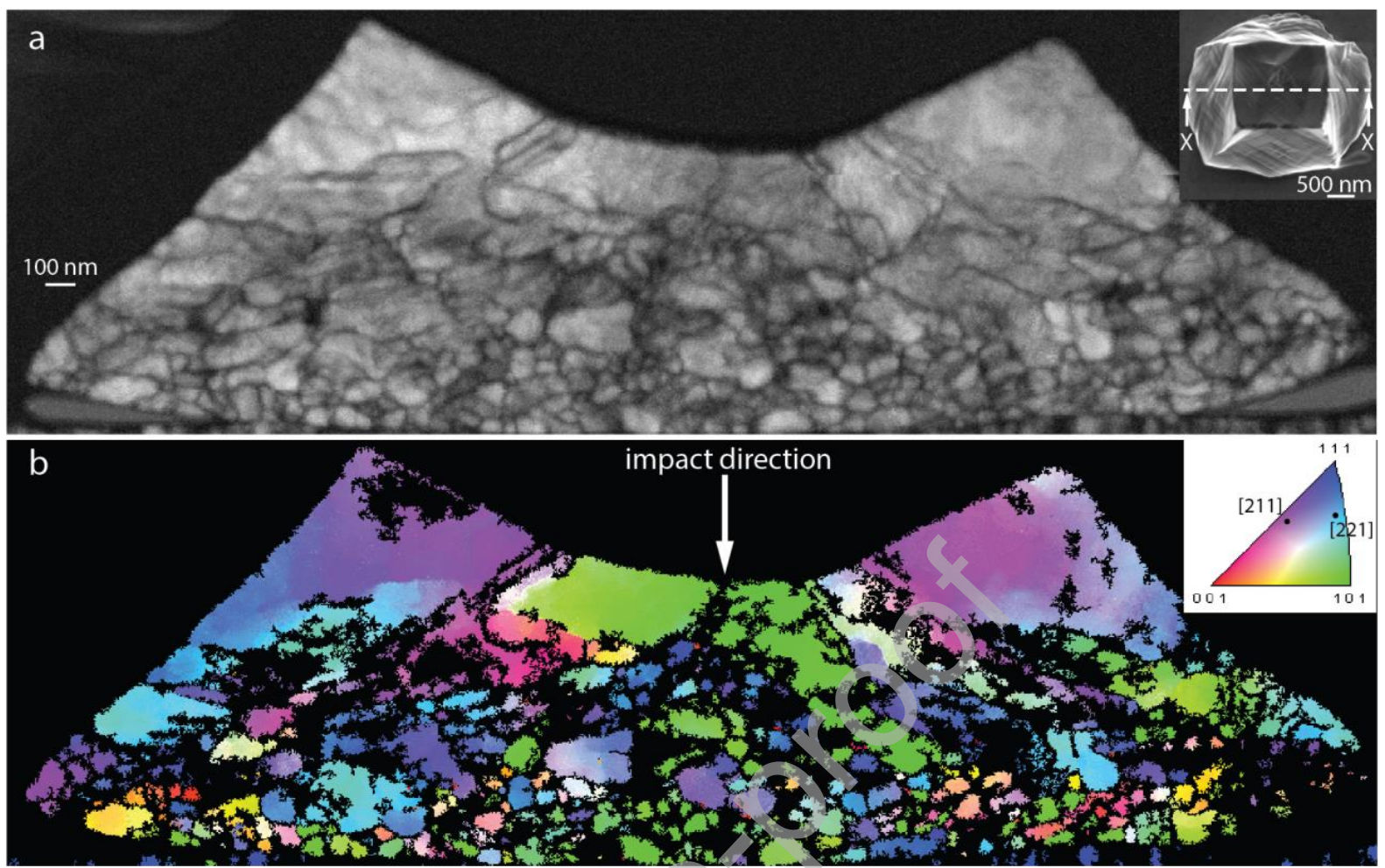

Figure 10. Post-recrystallization microstructure of an $\mathrm{Ag}$ microcube impacted along the [100] direction characterized by TKD technique: (a) Pattern quality map showing the GNG structure; inset shows the top view SEM of the impacted Ag microcube, (b) Orientation map (inverse pole figure color coded map) showing different grain orientations relative to the impact direction; inset shows the color scheme corresponding to different crystal symmetry directions.

\section{Conclusion}

Our observation of the $4 \mathrm{H}$ phase in impacted samples and its subsequent transformation back to the fcc phase through recrystallization over a week at room temperature shows intriguing solid-state crystallographic processes driven primarily by the stored mechanical energy and excess of vacancies from impact. The hcp phase in $\mathrm{Ag}$, particularly the $4 \mathrm{H}$ polytype has been shown to have high hardness, high resistance, less reflectivity, high surface plasmon absorption, Raman active mode, and low density $[25,26]$. The properties of the $2 \mathrm{H}$ phase are not well known yet. It may have potentially high catalytic activity due to its large inter-planar spacing between adjacent closepacked planes. Developing pathways to enhance stability of the new metastable phases - such as the demonstrated hcp phases in Ag-along with the unique GNG structure at ambient conditions by using solutes that can preferentially segregate to boundaries of those phases and nanograins [45] can 
potentially lead to unique metallic alloys with unprecedented functional properties that are desirable for engineering applications.

\section{Additional Information}

A supplementary information document and two supplementary videos that accompany the paper are available online. The source data of the figures can be obtained from the authors.

\section{Acknowledgements}

We acknowledge the financial support from the School of Engineering of the Rice University and the William and Stephanie Sick Chair of Engineering. RT and CG acknowledge the financial support from the University of Wisconsin-Madison. MP acknowledges the support from NSERC through the Discovery Grant (RGPIN-2016-06114) and Compute Canada. SJ acknowledges the financial support from the Ministry of Science, ICT, Korea, under the Information Technology Research Center support program (IITP-2018-2014-100639) supervised by the Institute for Information \& communications Technology Promotion. The support from the Electron Microscopy Center of the Rice University and the Center for Electron Nanoscopy of the Technical University of Denmark are acknowledged. We also thank Huifang Xu, Paul Evans, and Susan Babcock of the University of Wisconsin-Madison for the useful discussions.

\section{References}

[1] Y. Song, X. Chen, V. Dabade, T.W. Shield, R.D. James, Enhanced reversibility and unusual microstructure of a phase-transforming material, Nature. 502 (2013) 85-88. doi:10.1038/nature12532.

[2] K. Bhattacharya, Microstructure of Martensite: Why it Forms and how it Gives Rise to the Shape-memory Effect, OUP Oxford, 2003.

[3] P. Chowdhury, D. Canadinc, H. Sehitoglu, On deformation behavior of Fe-Mn based structural alloys, Mater. Sci. Eng. R Reports. 122 (2017) 1-28. doi:10.1016/j.mser.2017.09.002.

[4] J. Xing, F. Yuan, X. Wu, Enhanced quasi-static and dynamic shear properties by heterogeneous gradient and lamella structures in 301 stainless steels, Mater. Sci. Eng. A. 
680 (2017) 305-316. doi:10.1016/j.msea.2016.10.111.

[5] M. Miyahara, E. Ohtani, A. El Goresy, S. Ozawa, P. Gillet, Phase transition processes of olivine in the shocked Martian meteorite Tissint: Clues to origin of ringwoodite-, bridgmanite- and magnesiowüstite-bearing assemblages, Phys. Earth Planet. Inter. 259 (2016) 18-28. doi:https://doi.org/10.1016/j.pepi.2016.08.006.

[6] Z. Robert, T. Ryota, Y.M. L., S. Alan, F. Yasubumi, T. Sigurd, M. Burkhard, R. Mustafa, F. Jan, B. Hayo, C.Y. S., S. Vijay, J.R. D., T. Ichiro, E. Gunther, L. Alfred, Identification of Quaternary Shape Memory Alloys with Near- Zero Thermal Hysteresis and Unprecedented Functional Stability, Adv. Funct. Mater. 20 (2010) 1917-1923. doi:10.1002/adfm.200902336.

[7] D.J. Erskine, W.J. Nellis, Shock induced martensitic phase transformation of oriented graphite to diamond, Nature. 349 (1991) 317-319.

[8] R. Abeyaratne, J.K. Knowles, On a shock-induced martensitic phase transition, J. Appl. Phys. 87 (2000) 1123-1134. doi:10.1063/1.371989.

[9] A. Onodera, K. Inoue, H. Yoshihara, H. Nakae, T. Matsuda, T. Hirai, Synthesis of cubic boron nitride from rhombohedral form under high static pressure, J. Mater. Sci. 25 (1990) 4279-4284. doi:10.1007/BF00581085.

[10] B. Li, X. Wen, R. Li, Z. Wang, P.G. Clem, H. Fan, Stress-induced phase transformation and optical coupling of silver nanoparticle superlattices into mechanically stable nanowires, Nat. Commun, 5 (2014) 1-7. doi:10.1038/ncomms5179.

[11] D.R. Jones, B.M. Morrow, C.P. Trujillo, G.T. Gray, E.K. Cerreta, The $\alpha-\omega$ Phase transition in shock-loaded titanium, J. Appl. Phys. 122 (2017) 1-7. doi:10.1063/1,4987146.

[12] L.L. Hsiung, On the micromechanisms of shock-induced martensitic transformation in tantalum, AIP Conf. Proc. 845 I (2006) 228-231. doi:10.1063/1.2263305.

[13] M. Grouchko, I. Popov, V. Uvarov, S. Magdassi, A. Kamyshny, Coalescence of silver nanoparticles at room temperature: Unusual crystal structure transformation and dendrite formation induced by self-assembly, Langmuir. 25 (2009) 2501-2503. doi:10.1021/la803843k.

[14] D.R. Smith, F.R. Fickett, Low-temperature properties of silver, J. Res. Natl. Inst. Stand. Technol. 100 (1995) 119. doi:10.6028/jres.100.012. 
[15] F.S. Ameer, S. Varahagiri, D.W. Benza, D.R. Willett, Y.-M. Wen, F. Wang, G. Chumanov, J.N. Anker, Tuning Localized Surface Plasmon Resonance Wavelengths of Silver Nanoparticles by Mechanical Deformation, J. Phys. Chem. C. (2016). doi:10.1021/acs.jpcc.6b02169.

[16] E.F. Borra, O. Seddiki, R. Angel, D. Eisenstein, P. Hickson, K.R. Seddon, S.P. Worden, Deposition of metal films on an ionic liquid as a basis for a lunar telescope, Nature. 447 (2007) 979-981.

[17] M.I. Novgorodovo, A.I. Gorshkov, A. V. Mokhov, Native Silver and Its New Structural Modifications, Int. Geol. Rev. 23 (1981) 485-494. doi:10.1080/00206818109455083.

[18] E. Wetli, M. Hochstrasser, M. Erbudak, Epitaxial growth of Ag in the hexagonal structure, Surf. Sci. 377-379 (1997) 876-881. doi:10.1016/S0039-6028(96)01502-6.

[19] P. Taneja, R. Banerjee, P. Ayyub, G.K. Dey, Observation of a hexagonal (4H) phase in nanocrystalline silver, Phys. Rev. B. 64 (2001) 5-8. doi:10.1103/PhysRevB.64.033405.

[20] X. Liu, J. Luo, J. Zhu, Size effect on the crystal structure of silver nanowires, Nano Lett. 6 (2006) 408-412. doi:10.1021/n1052219n.

[21] A. Singh, A. Ghosh, Stabilizing high-energy crystal structure in silver nanowires with underpotential electrochemistry, J. Phys. Chem. C. 112 (2008) 3460-3463. doi:10.1021/jp7117967.

[22] B. Wang, G.T. Fei, Y. Zhou, B. Wu, X. Zhu, L. Zhang, Controlled growth and phase transition of silver nanowires with dense lengthwise twins and stacking faults, Cryst. Growth Des. 8 (2008) 3073-3076.

[23] C. Liang, K. Terabe, T. Hasegawa, M. Aono, Formation of metastable silver nanowires of hexagonal structure and their structural transformation under electron beam irradiation, Japanese J. Appl. Physics, Part 1 Regul. Pap. Short Notes Rev. Pap. 45 (2006) 6046-6048. doi:10.1143/JJAP.45.6046.

[24] Z. Fan, M. Bosman, X. Huang, D. Huang, Y. Yu, K.P. Ong, Y.A. Akimov, L. Wu, B. Li, J. Wu, Y. Huang, Q. Liu, C. Eng Png, C. Lip Gan, P. Yang, H. Zhang, Stabilization of 4H hexagonal phase in gold nanoribbons, Nat. Commun. 6 (2015) 7684. doi:10.1038/ncomms8684.

[25] I. Chakraborty, D. Carvalho, S.N. Shirodkar, S. Lahiri, S. Bhattacharyya, R. Banerjee, U. Waghmare, P. Ayyub, Novel hexagonal polytypes of silver: Growth, characterization and 
first-principles calculations, J. Phys. Condens. Matter. 23 (2011). doi:10.1088/09538984/23/32/325401.

[26] I. Chakraborty, S.N. Shirodkar, S. Gohil, U. V. Waghmare, P. Ayyub, A stable, quasi-2D modification of silver: Optical, electronic, vibrational and mechanical properties, and first principles calculations, J. Phys. Condens. Matter. 26 (2014). doi:10.1088/0953$8984 / 26 / 2 / 025402$.

[27] I. Chakraborty, S.N. Shirodkar, S. Gohil, U. V. Waghmare, P. Ayyub, The nature of the structural phase transition from the hexagonal $(4 \mathrm{H})$ phase to the cubic $(3 \mathrm{C})$ phase of silver, J. Phys. Condens. Matter. 26 (2014). doi:10.1088/0953-8984/26/11/115405.

[28] S.J. Jeon, S. Yazdi, R. Thevamaran, E.L. Thomas, Synthesis of monodisperse single crystalline Ag microcubes via seed-mediated growth, Cryst. Growth Des. 17 (2017) 284289. doi:10.1021/acs.cgd.6b01523.

[29] S. Jeon, J. Lee, E.L. Thomas, Polyol synthesis of silver nanocubes via moderate control of the reaction atmosphere, J. Colloid Interface Sci. 435 (2014) 105-111. doi:10.1016/j.jcis.2014.08.039.

[30] J.-H. Lee, P.E. Loya, J. Lou, E.L. Thomas, Dynamic mechanical behavior of multilayer graphene via supersonic projectile penetration., Science. 346 (2014) 1092-6. doi:10.1126/science.1258544.

[31] R. Thevamaran, O. Lawal, S. Yazdi, S.-J. Jeon, J.-H. Lee, E.L. Thomas, Dynamic creation and evolution of gradient nanostructure in single-crystal metallic microcubes, Science. 354 (2016) 312-316. doi:10.1126/science.aag1768.

[32] T.H. Fang, W.L. Li, N.R. Tao, K. Lu, Revealing extraordinary intrinsic tensile plasticity in gradient nano-grained copper., Science. 331 (2011) 1587-90. doi:10.1126/science.1200177.

[33] K. Lu, Making strong nanomaterials ductile with gradients, Science (80-. ). 345 (2014) 1455-1456. doi:10.1126/science.1255940.

[34] X. Wu, P. Jiang, L. Chen, F. Yuan, Y.T. Zhu, Extraordinary strain hardening by gradient structure., Proc. Natl. Acad. Sci. U. S. A. 111 (2014) 7197-201. doi:10.1073/pnas.1324069111.

[35] F. Niessen, A. Burrows, A.B. da S. Fanta, A systematic comparison of on-axis and offaxis transmission Kikuchi diffraction, Ultramicroscopy. 186 (2018) 158-170. 
doi:https://doi.org/10.1016/j.ultramic.2017.12.017.

[36] S. Plimpton, Fast Parallel Algorithms for Short-Range Molecular Dynamics, J. Comput. Phys. 117 (1995) 1-19. doi:https://doi.org/10.1006/jcph.1995.1039.

[37] M.P. Allen, D.J. Tildesley, Computer Simulation of Liquids, Clarendon Press, New York, NY, USA, 1989.

[38] P.L. Williams, Y. Mishin, J.C. Hamilton, An embedded-atom potential for the $\mathrm{Cu}-\mathrm{Ag}$ system, Model. Simul. Mater. Sci. Eng. 14 (2006) 817.

[39] M.W. Ullah, M. Ponga, A new approach for electronic heat conduction in molecular dynamics simulations, Model. Simul. Mater. Sci. Eng. 27 (2019) 075008. doi:10.1088/1361-651x/ab309f.

[40] L. Clément, R. Pantel, L.F.T. Kwakman, J.L. Rouvière, Strain measurements by convergent-beam electron diffraction: The importance of stress relaxation in lamella preparations, Appl. Phys. Lett. 85 (2004) 651-653. doi:10.1063/1.1774275.

[41] A. Stukowski, Structure identification methods for atomistic simulations of crystalline materials, Model. Simul. Mater. Sci. Eng. 20 (2012) 45021.

[42] E.F. Oliveira, P.A. da Silva Autreto, D.S. Galvão, On hardening silver nanocubes by highvelocity impacts: a fully atomistic molecular dynamics investigation, J. Mater. Sci. (2018). doi:10.1007/s10853-018-2104-z.

[43] T. V. Chitrakar, J.W. Keto, M.F. Becker, D. Kovar, Particle deposition and deformation from high speed impaction of Ag nanoparticles, Acta Mater. 135 (2017) 252-262. doi:10.1016/j.actamat.2017.05.062.

[44] W. Triftshäuser, J.D. McGervey, Monovacancy formation energy in copper, silver, and gold by positron annihilation, Appl. Phys. 6 (1975) 177-180. doi:10.1007/BF00883748.

[45] T. Chookajorn, H.A. Murdoch, C.A. Schuh, Design of Stable Nanocrystalline Alloys, Science. 337 (2012) 951-954. doi:10.1126/science.1224737. 


\section{Graphical Abstract}
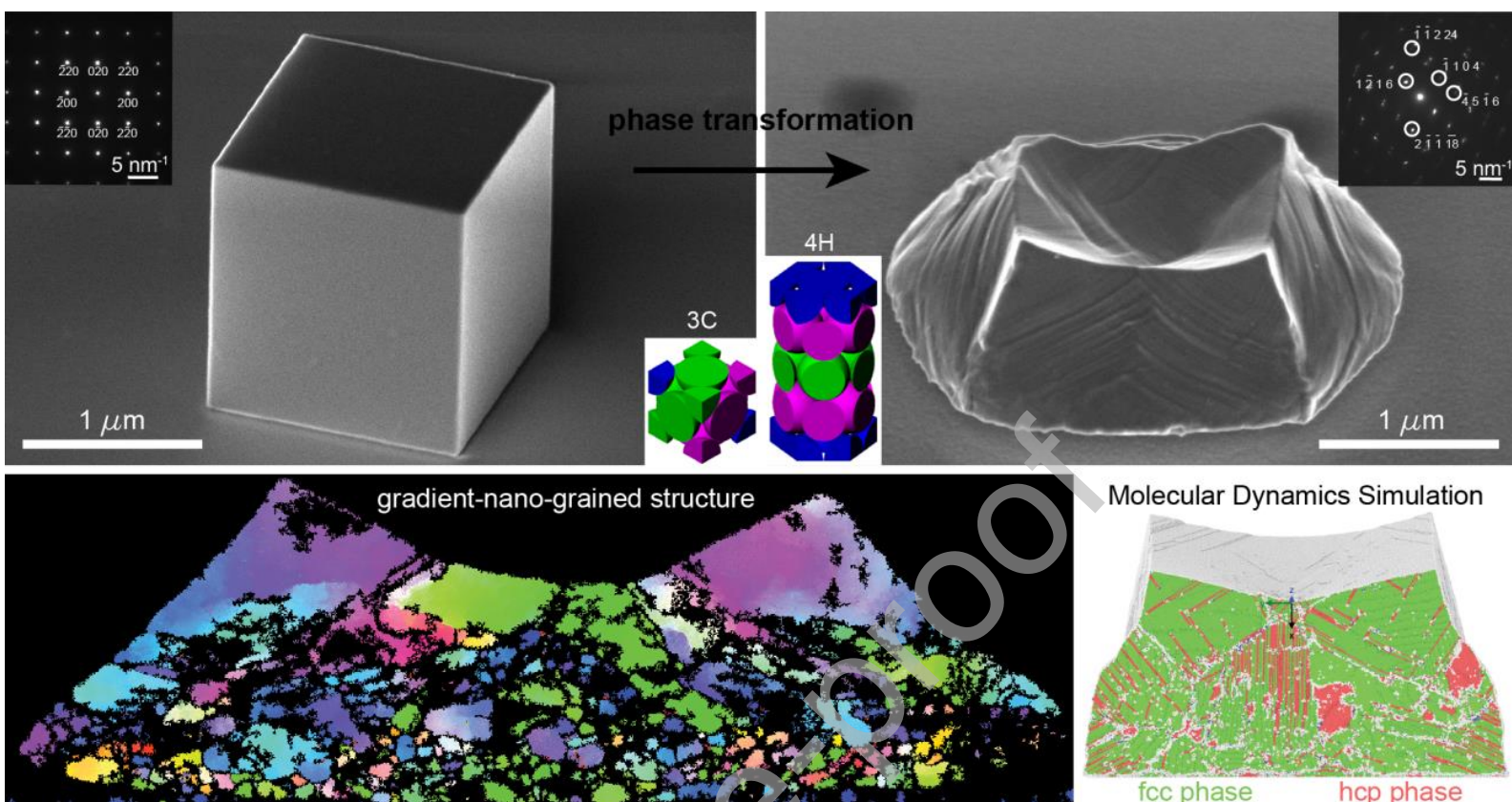

Molecular Dynamics Simulation

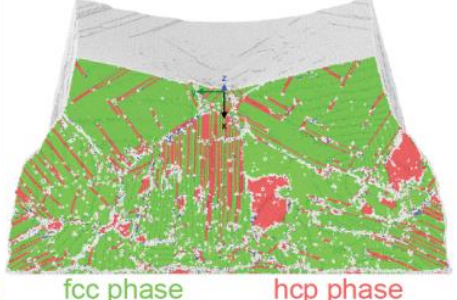

